\title{
The "why" and "how" of JointICA: Results from a visual detection task
}

\author{
Bogdan Mijović ${ }^{a, b, *}$, Katrien Vanderperren ${ }^{\mathrm{a}, \mathrm{b}}$, Nikolay Novitskiy ${ }^{\mathrm{c}}$, Bart Vanrumste ${ }^{\mathrm{a}, \mathrm{d}}$, Peter Stiers ${ }^{\mathrm{e}}$, \\ Bea Van den Bergh ${ }^{\text {f,g,h }}$, Lieven Lagae ${ }^{\mathrm{i}}$, Stefan Sunaert ${ }^{\mathrm{j}}$, Johan Wagemans ${ }^{\mathrm{c}}$, \\ Sabine Van Huffel ${ }^{\mathrm{a}, \mathrm{b}}$, Maarten De Vos ${ }^{\mathrm{a}, \mathrm{b}, \mathrm{k}}$ \\ ${ }^{a}$ Katholieke Universiteit Leuven, Department of Electrical Engineering, ESAT-SCD, Leuven, Belgium \\ b IBBT-K.U.Leuven Future Health Department, Leuven, Belgium \\ ${ }^{\text {c } K a t h o l i e k e ~ U n i v e r s i t e i t ~ L e u v e n, ~ L a b o r a t o r y ~ o f ~ E x p e r i m e n t a l ~ P s y c h o l o g y, ~ L e u v e n, ~ B e l g i u m ~}$ \\ ${ }^{\mathrm{d}}$ Katholieke Hogeschool Kempen, Biosciences and Technology Department, MOBILAB, Geel, Belgium \\ e Maastricht University, Faculty of Psychology and Neuroscience, Maastricht, The Netherlands \\ ${ }^{\mathrm{f}}$ Katholieke Universiteit Leuven, Department of Psychology, Leuven, Belgium \\ g Tilburg University, Department of Psychology, Tilburg, The Netherlands \\ h Flemish Government, Department of Welfare, Public Health and Family, Brussels, Belgium \\ ${ }^{\text {i }}$ Katholieke Universiteit Leuven, Department of Pediatric Neurology, Leuven, Belgium \\ ${ }^{j}$ Katholieke Universiteit Leuven, Department of Radiology, Leuven, Belgium \\ k Oldenburg University, Department of Psychology, Neuropsychology Lab, Oldenburg, Germany
}

\section{A R T I C L E I N F O}

\section{Article history:}

Received 18 October 2011

Revised 14 December 2011

Accepted 8 January 2012

Available online 16 January 2012

\section{Keywords:}

JointICA

EEG-fMRI

Data-fusion

Multimodal integration

Robustness

Validation

\begin{abstract}
A B S T R A C T
Since several years, neuroscience research started to focus on multimodal approaches. One such multimodal approach is the combination of electroencephalography (EEG) and functional magnetic resonance imaging (fMRI). However, no standard integration procedure has been established so far. One promising datadriven approach consists of a joint decomposition of event-related potentials (ERPs) and fMRI maps derived from the response to a particular stimulus. Such an algorithm (joint independent component analysis or JointICA) has recently been proposed by Calhoun et al. (2006). This method provides sources with both a fine spatial and temporal resolution, and has shown to provide meaningful results. However, the algorithm's performance has not been fully characterized yet, and no procedure has been proposed to assess the quality of the decomposition. In this paper, we therefore try to answer why and how JointICA works. We show the performance of the algorithm on data obtained in a visual detection task, and compare the performance for EEG recorded simultaneously with fMRI data and for EEG recorded in a separate session (outside the scanner room). We perform several analyses in order to set the necessary conditions that lead to a sound decomposition, and to give additional insights for exploration in future studies. In that respect, we show how the algorithm behaves when different EEG electrodes are used and we test the robustness with respect to the number of subjects in the study. The performance of the algorithm in all the experiments is validated based on results from previous studies.
\end{abstract}

(c) 2012 Elsevier Inc. All rights reserved.

\section{Introduction}

\section{Simultaneous EEG-fMRI}

The activation of a particular brain area consists of the synchronized firing of a subpopulation of neurons in that area, involved in processing information or executing a particular task. This synchronized relevant neural firing can be measured with the electroencephalogram (EEG) as event-related potentials (ERP). The neural activity is additionally accompanied by a regional increase in cerebral blood flow. These regional cerebral blood flow changes can be measured

\footnotetext{
* Corresponding author at: Kasteelpark Arenberg 10 - Box 2446, B-3001 Leuven, Belgium. Fax: + 3216321970

E-mail address: bogdan.mijovic@esat.kuleuven.be (B. Mijović).
}

directly as the blood oxygenation level dependent (BOLD) signal with functional magnetic resonance imaging (fMRI).

Moreover, EEG and BOLD changes can be measured simultaneously to benefit from their complementary properties. EEG measures electrical responses with a millisecond precision, but does not provide a precise spatial localization of the underlying cortical activity, since the electrode position is limited to the scalp surface. fMRI, on the other hand, measures local changes in brain hemodynamics with a very good spatial precision. However, the hemodynamic response is a slow signal and one echo-planar image (EPI) is only acquired every few seconds. This is far below the brain reaction time to externally applied stimuli, thus making the analysis of detailed temporal brain changes during the reaction on external stimuli very difficult. Moreover, an additional advantage of simultaneous recordings is the full reproducibility of the recording environment for both modalities. This is especially 
important in cognitive studies in which habituation, learning processes, arousal state or attention mechanisms play a role.

For this reason, the simultaneous acquisition of both EEG and fMRI is getting more and more popular, as their complementarity can provide deeper insight into function and dysfunction of brain dynamics (Debener and De Vos, 2011; Mulert and Lemieux, 2010; Ullsperger and Debener, 2010). This advantage has already been exploited in numerous applications. For instance, the combination of EEG and fMRI allows localizing epileptic activity based on spike-triggered fMRI (Bénar, et al., 2006; Krakow et al., 2001; Lemieux et al., 2001; Seeck et al., 1998). Other possible applications are the study of ongoing brain rhythms (Goldman et al., 2000, 2002; Laufs et al., 2003; Moosmann et al., 2003) and cerebral activations during sleep (Czisch et al., 2002; Liebenthal et al., 2003; Schabus et al., 2007). Also the analysis of event-related brain responses based on multimodal information (e.g. Calhoun et al., 2006; Debener et al., 2005, 2006; Eichele et al., 2008; Moosmann et al., 2008; Mulert et al., 2004) becomes more and more popular.

\section{Integration approaches}

In recent years, several integration approaches have also been proposed. The earliest proposed methods were EEG-informed fMRI analysis (such as Debener et al., 2005; Mulert et al., 2008; Novitskiy et al., 2011) and fMRI-informed EEG analysis (Yang et al., 2010; De Martino et al., 2011; Lei et al., 2011). These approaches are asymmetric, meaning that one of the modalities is considered to be prior knowledge to improve the results in the other modality. In fMRIinformed EEG, the fMRI sources are used to improve the localization of the ERP generators. In EEG-informed fMRI, the ERP information is used to model the strength of the hemodynamic response based on single-trial ERP amplitude modulations, with the aim of localizing the modulation-related fMRI sources.

Data-driven signal processing techniques are well-established for processing EEG data (e.g. De Vos et al., 2007, 2010; Porcaro et al., 2010; Viola et al., 2009), and are getting more and more popular for analyzing fMRI data (in the temporal domain: McKeown and Sejnowski, 1998; McKeown et al., 1998; McKeown, 2000; and in the spatial domain: Calhoun et al., 2001; Calhoun and Pearlson, 2004). Although no standard methods have been established, they are increasingly used for exploiting advantages of combined EEG-fMRI measurements. Attractive methods for this purpose are blind source separation (BSS) algorithms, such as independent component analysis (ICA), and canonical correlation analysis (CCA) (Mulert and Lemieux, 2010; Ullsperger and Debener, 2010). The best-known ICA-based algorithms for integrated EEG-fMRI analysis are Parallel ICA (Eichele et al., 2008; Lei et al., 2010; Liu and Calhoun, 2007), and JointICA (Calhoun et al., 2006, 2009), which will now be explained in more detail. CCA-based methods are not the focus of this paper; we refer the reader to Correa et al. (2008, 2010), and for the generalizations of correlation-based methods, see MartinezMontes et al. (2004).

The Parallel ICA algorithm identifies components in both modalities separately, applying spatial ICA to fMRI data, and temporal ICA to single trial EEG data (Eichele et al., 2008). After extracting the independent components from both modalities, the corresponding components are identified based on correlations between trial-to-trial fluctuations in the temporal domain. Although the Parallel ICA algorithm shows a nice performance in data-fusion applications, the algorithm does not provide full data-fusion, since the modalities are not allowed to influence each other at the first stage of the decomposition.

A similar approach is followed by Lei et al. (2010) with the difference that, in their study, the components are linked in both the spatial and temporal domain using variational Bayesian techniques. In that way, the information of one modality can be used as prior for the analysis of the other one, thereby enhancing its spatial (for EEG) or temporal (for
fMRI) resolution. In both these approaches, the data were processed separately first, and connections were only identified afterwards.

A parallel approach which imposes constraints during the parallel decomposition is proposed by Liu and Calhoun (2007). Although this approach also implies separate decompositions for the two modalities, a relationship can be defined between the two mixing matrices (belonging to the two modalities). As an example, in that paper the correlation between the mixing vectors of the two matrices is maximized.

JointICA, on the other hand, identifies the independent components for both modalities simultaneously. To do this, it starts from ERP epochs in the temporal domain and fMRI activation maps in the spatial domain. In the original paper on JointICA (Calhoun et al., 2006), the method was applied to averaged ERP data from different participants. Although another possible application has been shown on single-trial simulation data by Moosmann et al. (2008), in this paper we will focus on the original algorithm on averaged data. The method assumes that the different wave components (peaks) of the ERP and the spatial components in a statistical brain activation map (activation sites) of the same stimulus co-vary, either because they are generated in the same brain region (Logothetis et al. (2001) showed that the BOLD response is highly correlated with the group neuronal activity of the same brain region), or because the BOLD active areas had participatory roles in ERP activity, without necessary being the source of a particular ERP wave. Hence, it is believed that ICA will be able to disentangle these components and connect the electrical activations (ERP peaks) to their corresponding chemical (BOLD) brain activation sites (Calhoun et al., 2006).

The hypothesis about this one-to-one relation between the physiological origin of both data sets and their statistical dependence, however, is not necessarily true, since there is no particular mathematical reason to ensure capturing different ERP peaks always together with their corresponding fMRI activation sites. First of all, spatial statistical independence among fMRI components alone cannot be justified in the sense that there is no physical reason for the spatial samples to correspond to different activity patterns with independent distributions (see Daubechies et al., 2009). Furthermore, the two signals (ERP and fMRI) are very different in nature from a signal processing point of view. As used in the JointICA algorithm in this work, ERP is a temporal signal (we use information only from one electrode, which will be explained further in the manuscript) and fMRI a spatial map (obtained from a General Linear Model - GLM analysis) without any temporal information and their probabilistic distribution functions are different. The reader should note here that generally it is also possible to use concatenated ERPs from multiple electrodes as an input to JointICA, as done in the simulation study by Moosmann et al. (2008), therefore obtaining spatio-temporal ERP information. However, in this work, only ERPs from a single electrode are used.

Although JointICA was shown to provide meaningful results with real data when introduced as a tool for integrated ERP and fMRI data analysis (Calhoun et al., 2006, 2009), the underlying ICA assumption that the ERP and FMRI information extracted in a single component are mutually dependent, and at the same time statistically independent among different components was not questioned. Also, the behavior and robustness of the algorithm were not investigated. For this reason, and because of a growing interest in the method for the integration of different multimodal datasets, also besides EEG-fMRI (Calhoun et al., 2010; Doñamayor et al., 2011; Franco et al., 2008; Xu et al., 2009), some more methodological concerns need to be explored.

\section{The present study}

In this paper, we therefore try to explore and validate the performance of JointICA and its central assumption that physiological linking between ERP and BOLD amplitudes drives the extraction of multimodal components despite their intrinsically different temporal and spatial nature. We also show that the ICA algorithm used in the 
JointICA method (Infomax - Bell and Sejnowski, 1995) plays a crucial role in this multimodal separation. To support this claim, we investigate the performance of JointICA when other well-known ICA algorithms are used (JADE - Cardoso and Souloumiac, 1993; and FastICA - Hyvärinen, 1998), and show the disadvantages of these algorithms compared to the performance of Infomax.

To validate the meaningfulness of the JointICA results, we used a simple and well-established visual detection task with known ERP components and fMRI activation sites. This task is well-suited for validation purposes, as consistent results have been repeatedly found in several earlier studies (Di Russo et al., 2003, 2005; Novitskiy et al., 2011). In the mentioned work, the dipole pair accounting for the $C 1$ ERP wave was found to lie in the calcarine cortex. The early P1 wave dipole was situated in the dorsal extrastriate cortex of the middle occipital gyrus, and the late P1 dipole was found in the ventral fusiform gyrus. The dipole that accounted for the early N1 ERP wave, was localized in the parietal lobe near the intraparietal sulcus.

It is hypothesized that a large part of brain activity is reflected both in EEG and fMRI modalities, and the link between them can be established. This we refer to as the central linking hypothesis. If we can find components that reflect both EEG and fMRI activity, it provides evidence to support the CL hypothesis. The contribution of the central linking hypothesis is evaluated first by randomly reassigning ERPs and fMRI activation maps over participants, thus destroying the amplitude link between the two modalities, and second by a comparison with an individual ICA analysis of both ERPs and fMRI maps, thus excluding any intermodal interaction. Furthermore, we investigate the effect of the ERP quality for JointICA by comparing recordings inside and outside of the scanner and the dependence of the JointICA results on the sample size.

Based on the results obtained with these different analyses we will discuss why and when (under which conditions) JointICA works well, hopefully providing a better understanding for potential future users.

\section{Materials and methods}

\section{Subjects}

Twenty-six healthy subjects (11 female and 15 male, aged 18-44) with no history of neurological or cardiological disorders participated in this study. Written informed consent was obtained in accordance with the local ethical committee guidelines. During the simultaneous measurements subjects were lying supine in the scanner on a cushion that ameliorated the pressure from the EEG electrodes on the head, and with soft cushions to the side to restrict head movement in the coil. Subjects were provided with earplugs and headphones to avoid any harmful effect from the fMRI acoustic noise. Eighteen out of these 26 subjects additionally participated in a session where only EEG was acquired outside of the scanner room with the same presentation paradigm. In the outside session, the subjects were seated in front of the computer screen. The visual angle was as close as possible to the one in the inside session. The refresh rate of the screen was $60 \mathrm{~Hz}$ in both cases. The computer room was dark, and there was no environmental noise during the task execution. The experimental circumstances were optimized such that they maximally correspond between the two (inside the scanner, and outside the scanner) conditions.

\section{Tasks}

Participants performed a simple visual detection task while in the scanner. Stimuli consisted of segments of circular black-and-white checkerboard stimuli presented one at a time in randomized sequences to one of the four quadrants of the visual field (Novitskiy et al., 2011). The duration of a presented stimulus was $150 \mathrm{~ms}$. A central stimulus was also present, but was not used for the purpose of this study. Subjects were asked to press a button upon detection of each of these stimuli. Per run 100 stimuli (20 of each type) were shown to the participants. The inter-stimulus interval (ISI) varied between $900 \mathrm{~ms}$ and $1900 \mathrm{~ms}$. In each acquisition block, 61 empty stimuli are included. This, together with the fact that there are 5 different kinds of stimuli, gives rise to effective ISI, as shown in Wager and Nichols (2003). The average appearance of the consecutive stimuli of the same type is $6.8 \mathrm{~s}$, taking into account that the central stimulus activations overlap with each of the other stimuli separately. The presentation paradigm was randomized across subjects, as well as across sessions (inside versus outside). A more detailed description of the task can be found in Novitskiy et al. (2011), where part of the same dataset was used. However, in the latter paper within subject trialto-trial variations are explored, whereas the current work focuses on the ERP related BOLD activations based on inter-subject variability.

\section{Data acquisition}

All subjects performed 4 runs of the visual detection task. For validation purposes the EEG data were not only acquired in the magnetic field of the scanner, but 18 out of 26 subjects also performed the same task outside the scanner room. The EEG data were collected from 62 standard scalp sites using the BrainAmp MR + system (BrainProducts, Munich, Germany) with a sampling rate of $5 \mathrm{kHz}$. All channels were recorded with $\mathrm{FCz}$ as reference and $\mathrm{Iz}$ as ground. Electrode impedances were kept below $10 \mathrm{k} \Omega$. fMRI data were recorded with a Philips $3 \mathrm{~T}$ Intera whole-body scanner. During each experimental block, 160 echo-planar images (EPI) composed of 28 slices of $3 \times 3 \times 4.5 \mathrm{~mm}$ voxel size and $4.8 \mathrm{~mm}$ slice thickness were recorded with ascending slice order and 1.95 second repetition time (TR). Time to echo was $33 \mathrm{~ms}$, and the flip angle was $90^{\circ}$. For anatomical reference, full brain anatomical images were obtained with the magnetization prepared rapid gradient echo (MPRAGE) imaging sequence (230 coronal slices, time to echo $[\mathrm{TE}]=4.6 \mathrm{~ms}, \mathrm{TR}=9.7 \mathrm{~s})$.

\section{EEG processing}

Preprocessing of the acquired EEG data was performed in the MATLAB 7.7.0 (R2008B) (The Mathworks Inc, Natick, Massachusetts, USA) environment with the EEGLAB 5.03 toolbox (Delorme and Makeig, 2004).

First, gradient artifacts were removed with the average template subtraction method (Allen et al., 2000), as implemented in the Bergen EEG-fMRI EEGLAB plug-in (Moosmann et al., 2009). After filtering and downsampling to $500 \mathrm{~Hz}$, ballistocardiogram (BCG) artifact removal was performed with the Optimal Basis Set (OBS) method (Niazy et al., 2005) by creating an artifact template with 3 principal components (as validated in Vanderperren et al., 2010). To create the ERPs, data were re-referenced to the average of TP9 and TP10 (the closest electrodes to the mastoids in the present electrode setup), segmented to $100 \mathrm{~ms}$ before until $500 \mathrm{~ms}$ after the stimulus onset, base-line corrected $(-100 \mathrm{~ms}-0)$ and thresholded at $150 \mu \mathrm{V}$. Thereafter, an average ERP for each stimulus type was computed, and these averaged ERPs were further fed into the JointICA analysis (see below). The EEG data recorded outside the scanner were only filtered and downsampled and averaged ERPs were created in the same way as explained above.

For the application of the JointICA algorithm, only the ERP data from the electrodes PO7 and PO8 were used to observe the activations corresponding to right and left visual fields respectively (one electrode per application of the method). The ERP belonging to the $\mathrm{Oz}$ electrode was additionally used because the C1 ERP peak, corresponding to the activations in the primary visual cortex, is observable only on the midline electrodes (Di Russo et al., 2003, 2005; Martinez et al., 2001). 


\section{fMRI processing}

fMRI analysis was performed with the statistical parametric mapping software (SPM5, Wellcome Department of Cognitive Neurology, London, UK) in MATLAB. The EPI time series were slice-time corrected, realigned, co-registered with anatomical images, normalized to the MNI template and smoothed with an 8-mm FWHM Gaussian kernel.

A stick function at the onset of the stimulus, as in traditional event-related fMRI studies, was used as regressor. First-order statistics were calculated by convolving the stick function at the stimulus timing with a canonical hemodynamic response function (HRF), separately for the 5 stimulus locations (upper-left - UL, upper-right UR, lower-left - LL, lower-right - LR, and central stimulus - CC). No derivatives were used in the analysis. The 6 movement parameters (three for both translation and rotation) obtained during realignment were inserted in the model as covariates of no interest. As a result, the design consisted of $5+6=11$ variables per experimental block per subject. For each subject and condition, percent signal change (PSC) maps are then computed to be used in further analyses.

\section{JointICA}

JointICA (jICA) has originally been proposed by Calhoun et al. (2006). It is implemented in the Fusion ICA Toolbox, available from http://icatb.sourceforge.net. The JointICA algorithm assumes that the electrical correlate of brain activation (expressed in the ERP components) and the hemodynamic response to brain activation (BOLD response) are generated by the same population of neurons. Hence, the amplitudes of the ERP wave (peak) and of the BOLD response invoked by an activated area will increase and decrease synchronously: a stronger ERP peak activation will yield a stronger BOLD response in this particular brain region, and vice versa. Additionally, JointICA will reveal regions that were not directly involved in the creation of the ERP, but participated in the source activity, and therefore covariate strongly with a particular ERP wave.

Following this assumption, we can concatenate the PSC fMRI maps, derived from contrasting the BOLD signal invoked by a particular stimulus with the background, with the average ERP response corresponding to the same stimulus. Prior to this concatenation, several additional steps (listed below) are required, in order to avoid the bias to a particular modality.

First, to compensate for the disproportion between the number of voxels in the fMRI map and the number of time samples in the ERP signal, the ERP data are upsampled using cubic spline interpolation. After upsampling, the data of the two modalities are normalized (divided by the root mean squared data of all subjects) and concatenated into a single vector for each subject. The data from all the $m$ subjects are then stacked into an $m$ by $n$ matrix ( $m$ being the number of subjects, $n$ the number of samples of our artificially created data), and finally fed into an ICA algorithm to solve the following linear mixing problem:

$$
\left[\mathbf{X}^{\mathrm{fMRI}} \mathbf{X}^{\mathrm{EEG}}\right]=\mathbf{A} \times\left[\mathbf{s}^{\mathrm{fMRI}} \mathbf{s}^{\mathrm{EEG}}\right]
$$

$\mathbf{X}$ and $\mathbf{s}$ represent the matrices of, respectively, acquired and source signals, with their superscripts indicating their correspondence to the fMRI or ERP matrix part. Eq. (1) assumes the same mixing matrix $\mathbf{A}$ for both modalities. As mentioned before, this means that the corresponding parts of the ERP peaks and the BOLD responses of the corresponding brain regions are assumed to be changing with the same dynamics across subjects.

Following what has been mentioned previously, the JointICA algorithm provides, as an output, independent components, each containing the sources of both modalities (EEG and fMRI). In the Results section, these components are always shown in paired figures, one containing the EEG, and the other one the fMRI information.
Several ICA algorithms can be used in this setup. In this study we compare the results from the Information Maximization algorithm (Infomax), proposed by Bell and Sejnowski, (1995), Joint Approximate Diagonalization of Eigen-matrices (JADE; Cardoso and Souloumiac, 1993) and FastICA (Hyvärinen et al., 2001).

\section{ICASSO analysis}

ICASSO is a robustness analysis tool (Himberg and Hyvärinen, 2003; Himberg et al., 2004) for ICA results. ICA algorithms iteratively try to maximize a cost function, and are subject to the risk of instable solutions due to suboptimal local maxima. ICASSO assesses the stability of the obtained solution by running the same ICA algorithm starting from different initial points and with different bootstrapping, a number of times (in our work 30), and analyzes the differences between the multiple estimates of the independent components. ICASSO matches components across different runs by clustering them based on the absolute value of the correlation between squared source estimates. The clusters will be compact, if the correlations between different estimates of the same IC are very high. If, however, the correlation between different clusters is very high, one might consider performing an additional data reduction step, and repeat the analysis with a lower number of estimated independent components.

The algorithm also returns the stability index Iq for each estimated cluster. In the ideal case, the estimates are concentrated in mutually orthogonal clusters, in which case this index yields a value very close to 1 . If the clusters grow wider and start mixing, this value drops.

The ICASSO toolbox performs the FastICA algorithm. However, we additionally adapted it to use it with Infomax. The toolbox has already been used for the analysis of fMRI data (Correa et al., 2007).

The ICASSO toolbox itself can be found at http://research.ics.tkk.fi/ ica/icasso/.

\section{Evaluation methods}

\section{Application of JointICA - simultaneously recorded data}

To investigate the performance of JointICA, we start with applying it to the ERP and fMRI data recorded simultaneously. We visually validate the technique by comparing the activations obtained from JointICA when the PO8 electrode is used with what is established in the literature (Di Russo et al., 2003, 2005; Martinez et al., 2001).

According to the mentioned literature, primary visual areas are located around the calcarine sulcus, and correspond to the C1 ERP wave. However, this wave is difficult to be seen on the P08 electrode, but it is present on the midline electrodes $(\mathrm{Oz}, \mathrm{POz})$. The $\mathrm{C} 1$ peak for the DL stimulus is positive, and has a peak latency of around $100 \mathrm{~ms}$ (Martinez et al., 2001). The activations start between $65 \mathrm{~ms}$ and $90 \mathrm{~ms}$. Therefore, we performed JointICA also with the $\mathrm{Oz}$ electrode, and we verified resulting first brain activations for all 4 stimulus-types.

2. Application of JointICA - non-simultaneously recorded data The magnetic environment of the MRI scanner invokes severe artifacts in the recorded EEG signals that can only be removed at the risk of damaging the brain signals in the measurements. Both residual artifacts and damaged brain signals can interfere with the optimal performance of the JointICA analysis. To evaluate the magnitude of these unknown nuisance factors we also applied the JointICA analysis on EEG data free of the magnetic field artifacts and distortions.

3. JointICA experiment - investigating the influence of the ICA algorithm To more fully understand the performance of JointICA and to be able to explain how and why it works, we performed several additional analyses. To begin with, we tested the importance of the ICA algorithm used for JointICA. All the results reported so far were obtained with the Infomax algorithm (Bell and Sejnowski, 1995). We also tested the JointICA performance using two other well-known ICA algorithms: 
JADE (Cardoso and Souloumiac, 1993), which maximizes the statistical independence of the components by diagonalizing the 4th order cumulant of the mixing matrix, and FastICA (Hyvärinen et al., 2001), which uses a fixed point iteration scheme. FastICA extracts sources based on their kurtosis, as does JADE, but the convex optimization problem is solved using a cost function, instead of diagonalizing the kurtosis matrix.

4. JointICA experiment - central linking hypothesis and randomization To check the central linking hypothesis, we also applied the Infomax algorithm separately to ERP and fMRI data in order to show the difference for both modalities compared to the JointICA analysis on the simultaneously acquired data. Based on these analyses, we expect to understand whether the JointICA algorithm separates components and sources based on the interaction between both modalities, or whether the separation of one of the modalities is influenced (or driven) by the separation of the other one.

As explained before (see Eq. (1)), JointICA assumes that the average amplitudes of both EEG and fMRI responses have the same pattern across subjects. It is worth noting that the correlation between average ERP and BOLD amplitudes is also proposed by Bagshaw and Warbrick (2007).To verify this hypothesis, we connected random simultaneously acquired ERP and fMRI data (corresponding to different subjects) and applied the algorithm to check how the decomposition is affected. In this case, the amplitudes of ERP and fMRI responses are not expected to change synchronously, since they come from different subjects.

5. JointICA experiment - robustness and consistency analysis Finally, we investigated the robustness of the method. There were two aspects that needed to be checked in this respect: One was to check the consistency of the ICA decomposition. As mentioned in the previous section, the ICASSO toolbox was used for this purpose as to check the consistency of the Infomax and the FastICA decompositions. Another point to investigate was how the decomposition is dependent on the number of subjects in the study, and how it affects the decomposition when the number of participants falls below the suboptimal level. Therefore we applied a leave-oneout procedure, and checked the consistency for every number of subjects from 26 to 12 for the simultaneously recorded data, and from 18 to 12 for the data where the outside recorded EEG is used. The subjects were left out at random, but from 26 to 18, only the subjects without outside recordings were dropped, so that the subsets of 18 subjects for inside and outside recorded ERP data consist of the same participants.

The number of extracted independent components was 26 for the simultaneous measurements, and 18 for the non-simultaneously recorded data. The optimal model order was checked with the Akaike Information Criterion (AIC), the Minimum Description Length (MDL) and the R-index (a heuristic Davies-Bouldin type relative measure for a "natural" number of clusters, as implemented in ICASSO). All three methods estimated the information dimension (the number of components that should be extracted) equal to the full dataset dimension. This same estimate was also made for the EEG modality alone, whereas the dimensionality of the fMRI data was mostly estimated to be between 4 and 6 .

As a final check of the JointICA robustness performance, we masked a part of the fMRI data, and compared the results to the case where the values from the whole brain are used.

For all the additional analyses we will present and discuss the results only for the stimulus appearing in the down-left visual field, but the conclusions for other stimuli remain the same. Moreover, since we are interested in the fusion of fMRI and ERP modalities, and therefore only in the independent components which show both ERP and fMRI activation, we concentrate in this work only on ICs which contain $90 \%$ of the total ERP IC energy in the time range of $50-350 \mathrm{~ms}$.

In the following section (Results), in several places, correlation analysis is used to compare different independent components. In these comparisons, the corresponding independent components are matched based on the highest mutual correlation coefficient, i.e. the two independent components are corresponding if the correlation coefficient between these two is higher than if one of them is compared to any other one.

\section{Results}

\section{Application of JointICA - simultaneously recorded data}

In this section, we present the results of the analyses on data for the simultaneous measurements in two ways. In Fig. 2, the JointICA decomposition is shown for the down-left visual field stimuli. The ERP data were derived from the electrode PO8, on which the P1, N1, and P2 ERP peaks are clearly visible, and the corresponding fMRI IC activations obtained from JointICA are shown.

Additionally, since the C1 ERP peak reflecting the initial bottomup neural response of primary visual neurons located in the calcarine sulcus, is not visible on the more lateral PO8 electrode (Di Russo et al., 2003, 2005), Fig. 3 integrates the first activation results of all four stimuli in different colors, corresponding to the $\mathrm{C} 1$ peak IC of the $\mathrm{Oz}$ electrode. The detailed decomposition when the $\mathrm{Oz}$ electrode is used for the down-left stimulus is shown in Fig. 4.

Each subfigure in Figs. 2a-f presents a specific fMRI IC activation, and the corresponding grand average ERP, together with the ERP IC wave (shown in yellow and blue respectively). Since only the positive expected fMRI activations are available in the literature which we use for validation, only the positive fMRI IC activations are also shown in this study. The reader should note that Figs. $2 \mathrm{c}$ and d show the same independent component. They differ only in the slice that is taken to show the fMRI activations.

The locations of the fMRI IC activations in the brain are given in MNI coordinates in the figure captions, and anatomic areas are described. The P1 IC is associated with the activation in the right middle occipital gyrus (Brodmann Area 37). The N1 IC gives two components, the early one, associated with the right precuneus, cuneus and left lingual gyrus (BA 19, BA 7 and BA 18). During the late N1 ERP independent component, the areas in the right lateral occipital cortex (LOC) are active (BA 37). During this peak, also the activation in the somatosensory cortex (BA 3, 1 and 2) and primary motor cortex (BA 4) are visible. This activation is expected, since the subject was asked to press the button each time the stimulus appears. The component in Fig. 2e shows the ERP IC which appears as a transition from N1 to P2 ERP peak. This shows the activation in the right and left cuneus, and the right and left middle occipital gyrus. The P2 ERP peak is associated with the activations in the right middle occipital gyrus and right and left cuneus. These activations are expected, and are in line with previous findings in the literature (Di Russo et al., 2003, 2005; Martinez et al., 2001). It is interesting to note that the explained fMRI variance by the fMRI ICs shown in Fig. 2 was, $60 \%$, while another $20 \%$ is explained by the activations in the early visual areas which did not belong to any of the ERP IC waves (extracted with the ERP data on PO8).

To further investigate the quality of the JointICA decomposition, in Fig. 3 we present the first fMRI activations that emerge in the fMRI spatio-temporal map, which can be considered the beginning of the visual activation. These activations correspond to the C1 ERP IC of the $\mathrm{Oz}$ electrode. The ERPs for down-left (DL), down-right (DR), upper-left (UL) and upper-right (UR) stimuli are presented in different colors. The corresponding ERPs with their C1 independent component are shown in the corners coinciding with the position in the visual field where the particular stimulus was presented, in colors describing the corresponding fMRI activation.

Fig. 4 presents in detail all the activations derived with the ERP from the Oz electrode. Fig. 4a shows the fMRI IC activations around the calcarine sulcus that are associated with the C1 ERP independent component. The activated areas associated to the other ERP 
components (N1 early, N1 late and P2), correspond closely to the activations of the same ERP peaks, recorded on another electrode (PO8), shown in Fig. 2.

To verify the correspondence of the fMRI maps of Figs. 2 and 4, the correlations between the independent components are calculated. The correlation values are 0.8 for the early N1 independent component, 0.73 for the late $\mathrm{N} 1$ component and 0.8 for the P2 component. In contrast, the $\mathrm{C} 1$ associated fMRI IC from the $\mathrm{Oz}$ analysis (Fig. 4a) does not correlate with the P1 associated fMRI component from the PO8 analysis (Fig. 2a) $(r=-0.12)$. This confirms that these two fMRI IC activation maps are entirely different, despite the fact that the fMRI data entered into the two analyses were identical and only the different ERPs could have caused this different result.

\section{Application of JointICA - non-simultaneous recordings}

In addition to the simultaneous acquisition, a non-simultaneous EEG measurement was performed for 18 out of the 26 subjects, in which the EEG data were recorded during the scanner room, during the same task (Assecondi et al., 2010; Vanderperren et al., 2010). The grand average ERPs of the inside and outside sessions are being compared in Fig. 1. The difference between the inside and the outside acquired ERPs can be due to magnetic field artifacts, as well as due to different environmental settings, like the scanner noise, or the supine versus upright position. The differences between the two grand average ERPs were much lower (RMSE $=0.82$ ), than the individual average ERPs of the corresponding subjects (mean RMSE across subjects was 1.81 , with a minimum value of 0.97 and a maximal value of 3.1, see Fig. 1 for details).

To investigate the difference in quality between the data measured outside of the scanner room and the data measured inside the scanner, we computed the signal-to-noise ratios (SNRs) of the inside and the outside recorded ERPs for each subject. The SNR was computed such that the single trial ERP estimate was compared to the average ERP of the same subject. The provided SNRs are computed for the subset of 18 subjects, having both inside and outside recordings. The number of single trials in each average ERP was between 75 and 80 for both inside and outside recordings. The mean SNR for the inside data was $0.2484 \pm 0.0872$, whereas the SNR computed for the non-simultaneously measured data was $0.5385 \pm 0.2102$. The difference in the SNRs between the two datasets was significant $(\mathrm{p}<0.0001)$.

Taking into account the higher SNRs for the outside compared to the inside data, and the greater similarity of the GA compared to single subject averages, we argue that the residual magnetic field artifacts in the inside case do affect the ERP signals. Therefore, we propose that it is relevant to make use of the outside recorded data to assess the influence of the magnetic field artifacts on the JointICA.

Fig. 5 shows the results of applying the JointICA algorithm to the fMRI data from the simultaneous measurement combined with the
ERPs derived from the non-simultaneous measurement, again for the down-left stimulus condition. For an easier comparison, we overlaid the obtained fMRI ICs (blue) on top of the corresponding fMRI ICs obtained for the simultaneous measurements (red). The overlap between the two is shown in yellow.

From this figure, it can be seen that the fMRI IC activations for the inside and outside measurements, corresponding to the same ERP IC waves, largely overlap. As it was the case for the inside recorded EEG, contra-lateral fMRI activation is visible in the middle occipital gyrus connected to the P1 peak. Activations are also present in the right cuneus and precuneus, associated with the N1 early peak, and the active regions in somatosensory and motor areas coincide with the N1 late activity. Also the P2 peak is mostly associated with the activations in left and right cuneus.

The fMRI ICs resulting from the analysis with the $\mathrm{Oz}$ electrode measurements outside the scanner were also similar to the ones obtained in the corresponding analysis with the simultaneously recorded data (presented in Figs. 3 and 4). Therefore, these pictures are not additionally shown.

\section{JointICA experiment - investigating the influence of the ICA algorithm}

In Table 1, the cross-correlations between the independent components extracted by Infomax and FastICA (the first row) and Infomax and JADE (the second row) are shown. It is obvious that Infomax and FastICA yield similar components, with a mean cross-correlation value of $0.9815 \pm 0.5811$, whereas the results for Infomax and JADE are significantly different (mean correlation value of $0.7471 \pm 0.3736$, p-value of unpaired $t$-test $<0.0001$ ). The reported averages and standard deviations of the correlation values are corrected with the Fisher z-transformation throughout the manuscript.

Looking at the activations, the results from Infomax and JADE are different in the following sense: first, JADE did not yield specific P1connected visual components. Second, JADE combined the P1 and early N1 ERP waves together in one component, but also their corresponding fMRI activations were combined in this same component. Third, the independent component revealing the late N1 peak in the ERP modality did neither show motor nor N1-associated visual activation. Instead, it yielded activation that was expected to belong to the P1 peak. The rest of the activations were similar.

\section{JointICA experiment - central linking hypothesis and randomization}

Further, we compared the ERP and fMRI decompositions obtained with the JointICA analysis, to those obtained by applying Infomax to solely fMRI or inside and outside recorded ERP data. The correlations of the Infomax decomposition of the ERP and fMRI data obtained in separate and joint decompositions, corresponding to the down-left stimulus are shown in Table 2. The correlations between corresponding components from bi-modality and mono-modality analyses were
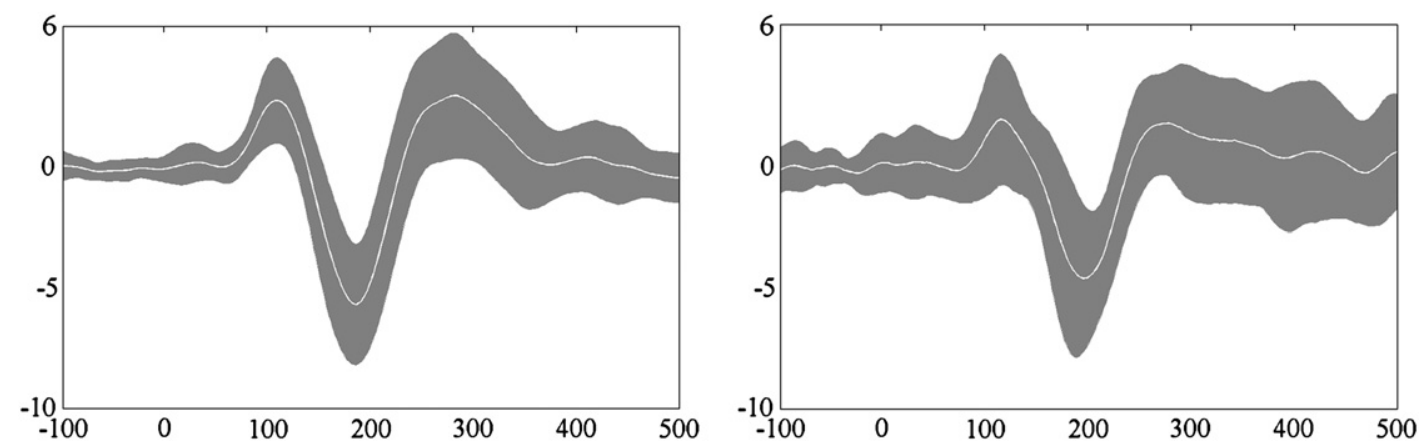

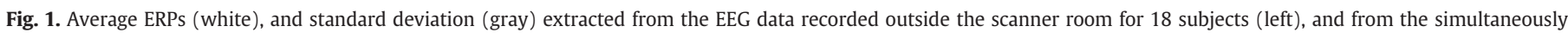
recorded EEG data for 26 subjects (right). The data are recorded on the PO8 electrode. 
Table 1

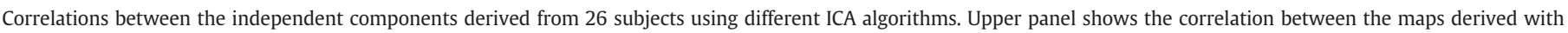

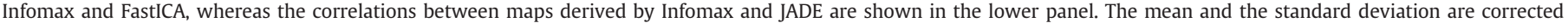
with the Fisher z-transformation.

\begin{tabular}{|c|c|c|c|c|c|c|c|c|c|c|c|}
\hline \multicolumn{6}{|c|}{ Infomax-FastICA correlation } & \multicolumn{6}{|c|}{ Infomax-JADE correlation } \\
\hline 0.9877 & 0.9913 & 0.9929 & 0.9723 & 0.8327 & 0.989 & 0.6886 & 0.4982 & 0.9573 & 0.9 & 0.7524 & 0.9065 \\
\hline 0.9924 & 0.9435 & 0.9927 & 0.9603 & 0.8767 & 0.9969 & 0.8633 & 0.7468 & 0.904 & 0.4829 & 0.7246 & 0.5808 \\
\hline 0.9901 & 0.9572 & 0.9961 & 0.9949 & 0.9882 & 0.994 & 0.4245 & 0.8691 & 0.8338 & 0.6417 & 0.6168 & 0.549 \\
\hline 0.9965 & 0.7324 & 0.8976 & 0.9883 & 0.9252 & 0.9175 & 0.567 & 0.7786 & 0.5996 & 0.5846 & 0.9166 & 0.482 \\
\hline 0.9888 & 0.9927 & & & & & 0.7537 & 0.6556 & & & & \\
\hline \multicolumn{6}{|c|}{ Mean $=0.9815 \pm 0.5811$} & \multicolumn{6}{|c|}{ Mean $=0.7471 \pm 0.3736$} \\
\hline
\end{tabular}

significantly higher for the ERP only $(0.7541 \pm 0.3539)$ compared to the fMRI only analysis $(0.6065 \pm 0.2205)(p=0.0018)$ for the inside case. In the case of 18 subjects (outside data), still the ERP only data were more strongly correlated $(0.7414 \pm 0.2465)$, than the fMRI data $(0.6887 \pm 0.3177)$, but this difference was not significant.

To further investigate the importance of the central linking hypothesis, the bottom row of Table 3 shows the correlation coefficients between the fMRI IC maps derived in a regular way with simultaneously recorded ERPs, and the fMRI IC maps derived with the same ERP data, but now randomly assigned to the fMRI maps (i.e. the fMRI and the ERP data of the subjects do not correspond to each other). Obviously, the correlation coefficient values are rather low, indicating that the amplitude changes in the ERP and fMRI components are closely related.

\section{JointICA experiment - robustness and consistency analysis}

To explore the robustness we performed several analyses, and we additionally employed the ICASSO method as described in the Materials and methods section. The minimal Iq values are given in Table 5 for different numbers of subjects in the experiment (12 to 26 for the simultaneous, and 12 to 18 for the non-simultaneous measurements). Fig. 6 presents the results of the ICASSO analysis for different setups. The red and pink lines respectively describe stronger and weaker connections between the estimated clusters. If the decomposition is consistent, the between-cluster connections do not exist.

First, ICASSO showed that the decomposition for both the 26 inside recorded subjects, and 18 subjects where the ERPs were recorded in separate sessions was stable, with the smallest Iq values of 0.9989 and 0.9536 , respectively (Figs. 6a-b). However, the ICASSO study provides only the information about the consistency of the decomposition itself, not about how is it influenced with respect to a different number of subjects. Therefore, we additionally checked the behavior of the decomposition when the number of subjects for the simultaneous measurement drops from 26 to 18. In this leaving-one-out procedure, always subjects without outside recording were left out, ending with the 18 subjects which have both inside and outside recordings. Besides this constraint, the subjects were left out completely at random. Since the difference between these two measurements is mostly reflected in the P1-related IC ERP and fMRI maps (see Figs. 2 and 5, and Table 4), we followed the behavior of the independent components, associated with this peak.

For the number of subjects being 25 , not much was changed (see Table 5). Then, the decomposition becomes unstable with 24 subjects (minimal Iq of 0.7982). Closer inspection of the joint decomposition, revealed that the $\mathrm{P} 1$ peak, which was alone in the decomposition for 26 subjects (Fig. 2), got separated in 2 peaks, but the fMRI activations were not interpretable. The decomposition is still unstable, until the number of subjects drops down to 19 (minimal Iq 0.9573). At that point, the two P1 peaks are merged together again, but with no interpretable fMRI component. The same holds with the 18 subjects, and this decomposition is shown in Fig. 7. These 18 subjects in Fig. 7 are the same subjects, whose outside results are shown in Fig. 5.

We additionally compared in Fig. 6 the robustness of the Infomax decomposition for 18 subjects recorded in the non-simultaneous and simultaneous sessions (Figs. 6b-c), and also the FastICA decomposition of the 18 subjects recorded in the simultaneous session (Fig. 6d). This figure shows that the Infomax inside decomposition for 26 subjects and the decomposition for 18 subjects recorded in the separate sessions are stable, meaning that they yield a constant decomposition, independently of the initialization of the Infomax algorithm (Figs. 6a-b). Fig. 6c shows that the Infomax decomposition of the simultaneous data is less robust than the one for the separate measurements, when an equal number of subjects (18) are used, with a minimal Iq of 0.8159 .

A comparison of the results obtained by Infomax and FastICA on the simultaneous measurements (Figs. $6 \mathrm{c}$ versus $\mathrm{d}$ ) shows that the FastICA algorithm is more unstable than Infomax (minimal Iq is only 0.6287). It is worth noting at this point that, when FastICA ICASSO is applied to the dataset of 26 subjects, it gives a very stable performance (Iq of 0.9932 ).

When the number of subjects is further reduced (the decomposition with 12 subjects is shown in Fig. 8), the components in the

Table 2

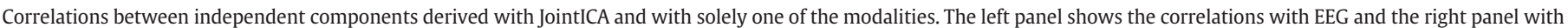
the fMRI modality. The mean and the standard deviation are corrected with the Fisher z-transformation.

\begin{tabular}{|c|c|c|c|c|c|c|c|c|c|c|}
\hline \multirow[b]{2}{*}{ Simultaneously recorded data } & \multicolumn{5}{|c|}{$\begin{array}{l}\text { Correlations of the ERP ICA to the ERP modality from JointICA } \\
\text { decomposition }\end{array}$} & \multicolumn{5}{|c|}{$\begin{array}{l}\text { Correlations of the fMRI ICA to the fMRI modality from JointICA } \\
\text { decomposition }\end{array}$} \\
\hline & 0.4281 & 0.5719 & 0.7727 & 0.7172 & 0.566 & 0.6260 & 0.6799 & 0.7633 & 0.4568 & 0.4744 \\
\hline & 0.3293 & 0.8916 & 0.5565 & 0.7468 & 0.6938 & 0.6177 & 0.3914 & 0.6591 & 0.4928 & 0.4957 \\
\hline & 0.8188 & 0.8726 & 0.8551 & 0.4571 & 0.9109 & 0.8107 & 0.524 & 0.6798 & 0.5974 & 0.4820 \\
\hline & 0.6024 & 0.3320 & 0.8689 & 0.8546 & 0.6907 & 0.8586 & 0.4682 & 0.4519 & 0.5587 & 0.7827 \\
\hline & 0.9249 & 0.8395 & 0.8351 & 0.8775 & 0.7544 & 0.6722 & 0.5638 & 0.595 & 0.3656 & 0.6578 \\
\hline & 0.7429 & & & & & 0.5850 & & & & \\
\hline & Mean $=$ & $41 \pm 0.35$ & & & & Mean $=$ & $65 \pm 0.22$ & & & \\
\hline \multirow[t]{5}{*}{ Nonsimultaneously recorded data } & 0.7053 & 0.5980 & 0.6991 & 0.7598 & 0.6902 & 0.7618 & 0.8742 & 0.4981 & 0.5728 & 0.8800 \\
\hline & 0.5234 & 0.6081 & 0.6119 & 0.6996 & 0.7680 & 0.4297 & 0.7714 & 0.4311 & 0.6687 & 0.8717 \\
\hline & 0.8513 & 0.7717 & 0.7822 & 0.9031 & 0.8655 & 0.7292 & 0.4785 & 0.5672 & 0.5179 & 0.4747 \\
\hline & 0.5925 & 0.7182 & 0.8572 & & & 0.8260 & 0.8244 & 0.5862 & & \\
\hline & \multicolumn{5}{|c|}{ Mean $=0.7414 \pm 0.2465$} & \multicolumn{5}{|c|}{ Mean $=0.6887 \pm 0.3177$} \\
\hline
\end{tabular}


Table 3

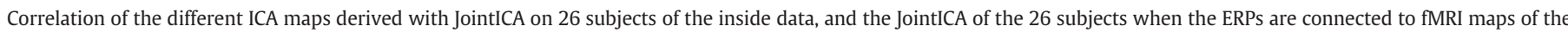
non-corresponding subjects. The mean and the standard deviation are corrected with the Fisher z-transformation.

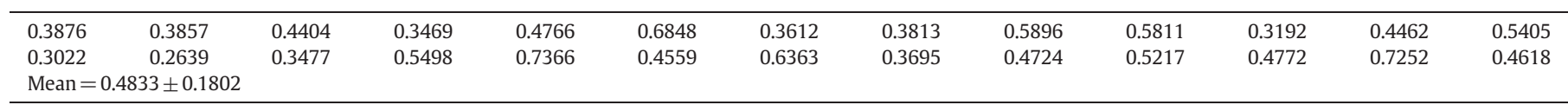

simultaneous Infomax setting are getting merged together, and the decomposition becomes stable again $(I q=0.9435$, see Table 5$)$. Note that in Table 5, we always leave the same subject out, in both inside and outside recordings. The subject which was to be left out is chosen completely at random.

Fig. 8a shows that the ERP IC merges the P1 and early N1 ERP peaks, but also the corresponding fMRI ICs are merged (when compared to Figs. 2a and b). These activations are presented in Fig. 8a in blue and green respectively. The overlaps with the decomposition with 12 subjects are presented in purple and yellow. Fig. 8b shows again the N1 late component, which shows the cuneus, precuneus, somatosensory and primary motor activation, same as Figs. 2c-d (shown in blue, overlap in purple). The same holds for Fig. 8c which shows the P2 ERP IC, and the corresponding fMRI IC maps, which correspond to the fMRI IC maps visible in Fig. $2 \mathrm{f}$ (shown in blue, overlap in purple).

It is also worth noting that for the non-simultaneous measurement, the Iq value is consistently high for all the number of subjects in the experiment from 12 to 18 , whereas considerable variability in this value is present for the simultaneously measured data (Table 5).
As a last step in the robustness analysis, we checked how the decomposition is affected by masking a part of the fMRI data. We applied a mask that only included the occipital area, together with the cuneus and precuneus areas (the region where we expected our activation, without somatosensory and motor areas). The results did not change in the sense that the same fMRI ICs were connected to the same ERP ICs. The only difference was that somatosensory and motor areas were no longer present in any of the components. Therefore, the late $\mathrm{N} 1$ peak, for example, contained only the visual activations, as shown in Fig. 2 d.

\section{Discussion}

In this study, we evaluated and presented the performance of JointICA, a recently proposed method for a symmetric integration of EEG and fMRI. To this end, we recorded EEG and fMRI data during a simple visual detection task. As this particular task is very well described in the literature (Di Russo et al., 2002, 2003, 2005; Martinez et al., 2001), we use this information to assess the relevance of the results in our experiments. For this reason, we did not additionally include

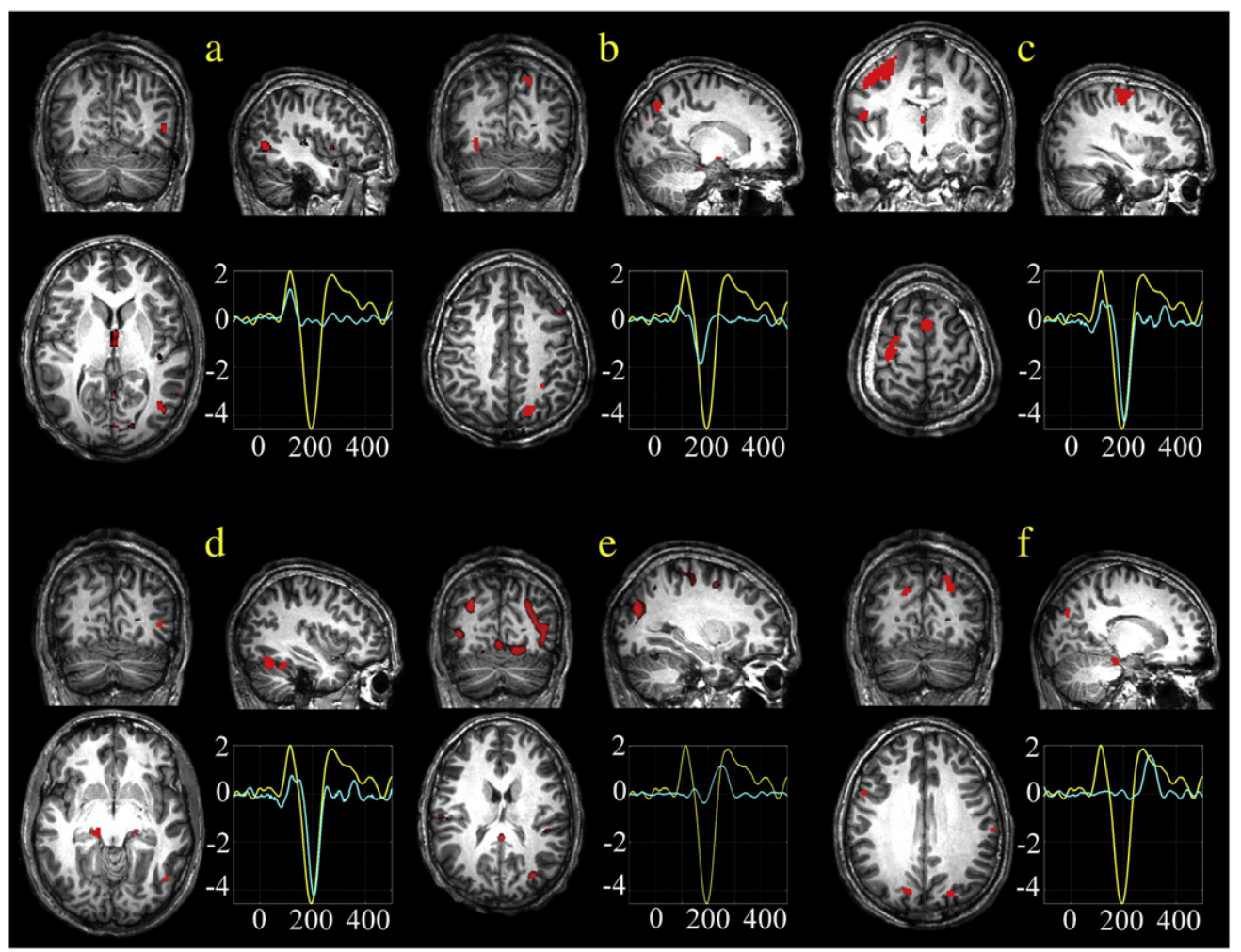

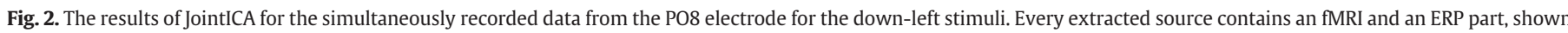

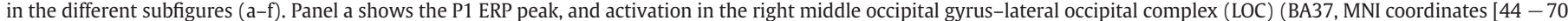

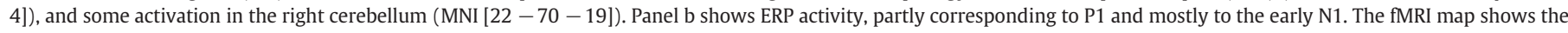

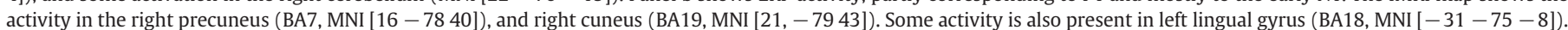

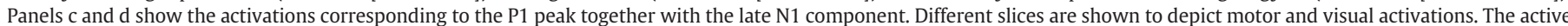

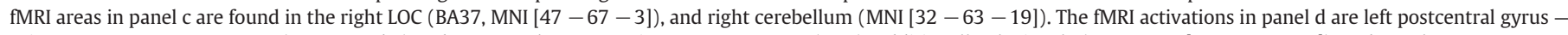

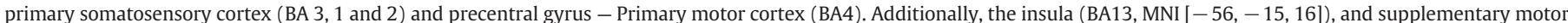

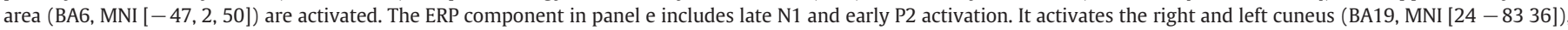

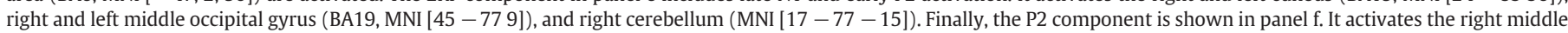
occipital gyrus (BA18, MNI [32 - 86 2]), right and left cuneus (BA7, MNI [24 - 83 36]) and right cerebellum (MNI [32 - 68 - 19]). 
Table 4

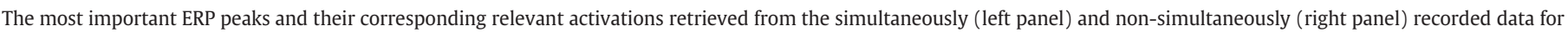

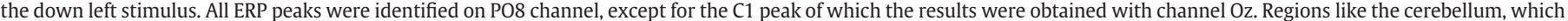

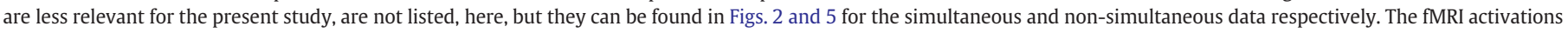
are presented with their anatomical region, and their corresponding Brodmann area (BA), and can be seen in Figs. 2 and 5 .

\begin{tabular}{|c|c|c|c|c|c|}
\hline \multicolumn{3}{|c|}{ Simultaneous data } & \multicolumn{3}{|c|}{ Non-simultaneous data } \\
\hline ERP peak & Anatomical region & Brodmann area & ERP peak & Anatomical region & Brodmann area \\
\hline $\mathrm{C} 1$ & Right upper lingual gyrus & BA17 & $\mathrm{C} 1$ & Right upper lingual gyrus & BA17 \\
\hline \multirow[t]{4}{*}{ P1 } & Right LOC & BA37 & P1 (early) & Right middle occipital gyrus & BA19 \\
\hline & & & & Right LOC & BA37 \\
\hline & & & & Left LOC & BA37 \\
\hline & & & P1 (late) & Right fusiform gyrus & BA19 \\
\hline \multirow[t]{3}{*}{ N1 (early) } & Right precuneus & BA7 & N1 (early) & Right precuneus & BA7 \\
\hline & Right cuneus & BA19 & & Right cuneus & BA19 \\
\hline & Left lingual gyrus & BA18 & & Right lingual gyrus & BA18 \\
\hline \multirow[t]{5}{*}{ N1 (late) } & Right LOC & BA37 & N1 (late) & Right middle occipital gyrus & BA19 \\
\hline & Left postcentral gyrus & BA $3,1,2$ & & Left poscentral gyrus & BA $3,1,2$ \\
\hline & Left precentral gyrus & BA4 & & Left precentral gyrus & BA4 \\
\hline & Left medial frontal gyrus & BA6 & & Left medial frontal gyrus & BA6 \\
\hline & Left Insula & BA13 & & Left Insula & BA13 \\
\hline \multirow[t]{3}{*}{ P2 } & Left cuneus & BA19 & $\mathrm{P} 2$ & Left cuneus & BA19 \\
\hline & Right cuneus & BA19 & & Right cuneus & BA19 \\
\hline & Right middle occipital gyrus & BA18 & & & \\
\hline
\end{tabular}

a simulation study but for simulation studies of the algorithms used in this paper, the reader is referred to Moosmann et al. (2008) and Daubechies et al. (2009).

We compared the performance of JointICA for data acquired both simultaneously and non-simultaneously and investigated whether the decomposition was equally driven by both modalities, or solely by one of them. Moreover, we examined the validity and necessity of the assumptions that JointICA (Calhoun et al., 2006) relies on the amount of available data and the selection of the ICA algorithm. Further, we investigated the influence of the robustness and consistency of the decomposition obtained with JointICA, validated the central linking theorem, and we proposed the necessary conditions for a reliable decomposition.

\section{Application of JointICA - simultaneously recorded data}

According to the literature and based on the characteristics of the presented task, the following activations are expected: the $\mathrm{C} 1$ peak should correspond to activity in the vicinity of the calcarine sulcus, whereas the early P1 peak is expected to activate the dorsal extrastriate areas of the middle occipital gyrus. Furthermore, the late P1 wave is known to be related to activations in the ventral fusiform gyrus,

\section{Table 5}

The effect on consistency of the derived independent components when the number of participants is dropping from 26 to 18 observed through the Iq measure derived with the ICASSO analysis. The $I q$ stands for quality (stability) index. The index in the table is the lowest stability index for the dataset consisted of a particular number of subjects.

\begin{tabular}{lll}
\hline $\begin{array}{l}\text { The number of } \\
\text { components }\end{array}$ & $\begin{array}{l}I q \text { (simultaneous } \\
\text { recordings) }\end{array}$ & $\begin{array}{l}\text { Iq (non-simultaneous } \\
\text { recordings) }\end{array}$ \\
\hline 26 & 0.9989 & - \\
25 & 0.9596 & - \\
24 & 0.7982 & - \\
23 & 0.7288 & - \\
22 & 0.6736 & - \\
21 & 0.8817 & - \\
20 & 0.7055 & - \\
19 & 0.9573 & - \\
18 & 0.8159 & 0.9506 \\
17 & 0.8964 & 0.9451 \\
16 & 0.9524 & 0.9445 \\
15 & 0.7565 & 0.9415 \\
14 & 0.9533 & 0.9403 \\
13 & 0.9518 & 0.9378 \\
12 & 0.9435 & 0.9453 \\
\hline
\end{tabular}

and the early N1 peak should yield activations of the parietal lobe, close to the intraparietal sulcus (Di Russo et al., 2002, 2003). In addition to this visual stimulation, our subjects were required to respond to the presented stimuli by pressing a button, which should evoke activation of the somatosensory and primary motor cortex.

The above expectations were consistently confirmed in our study both on simultaneously and non-simultaneously acquired data. However, as is shown in Fig. 2, the P1 ERP IC emerged as expected, but the activation connected to it was not complete in the sense that there was no activation in the ventral fusiform gyrus (as explained above).

Also in Figs. 3 and 4, we find the expected activations. It is clear that the $\mathrm{C} 1$ peak is positive for lower visual field stimuli, and negative for upper visual field stimuli. This comes from the fact that the activations which produce the C1 ERP peak lie on the opposite sides of the calcarine sulcus, and therefore their dipoles produce peak activations with an opposite polarity (Clark et al., 1995; Di Russo et al., 2002). It is also clear from Fig. 3, that the voxels associated with these early ERP components are located in or very close to the calcarine sulcus in the contra-lateral hemisphere of the brain, as expected. Also, voxels in the upper bank of the calcarine sulcus are associated with the $\mathrm{C} 1$ aspect of the ERPs invoked by the lower-half visual field stimuli and voxels in the lower bank of the calcarine sulcus are associated with this component from the upper-half visual field stimuli.

As a further argument that these decompositions are valid, we showed that the early components in the analyses with the PO8 electrode (Fig. 2) and the Oz electrode (Figs. 3 and 4) do not correspond. On the other hand, we showed that the later fMRI ICs from the $\mathrm{Oz}$ analysis are spatially highly correlated with the fMRI ICs from the PO8 analysis (also visible in Fig. 4).

\section{Application of JointICA - non-simultaneous recordings}

Making use of the EEG data measured outside of the scanner room, slightly improved the interpretability of the results and yielded spatio-temporal activations more consistent with the literature, specifically for the P1-related component. Earlier work by Martinez et al. (2001) showed that the P1 ERP wave is generated by two different regions. One region is found in the middle occipital gyrus (this activation is present in both inside and outside maps, Figs. 2a and 5a). However, another region that contributes to the late P1 activation is located in the fusiform gyrus. This contribution can be clearly seen in Fig. 5b, but not in Fig. 2a, nor in any other subfigure of Fig. 2. It is also worth noting that the locations of the brain activation clusters 


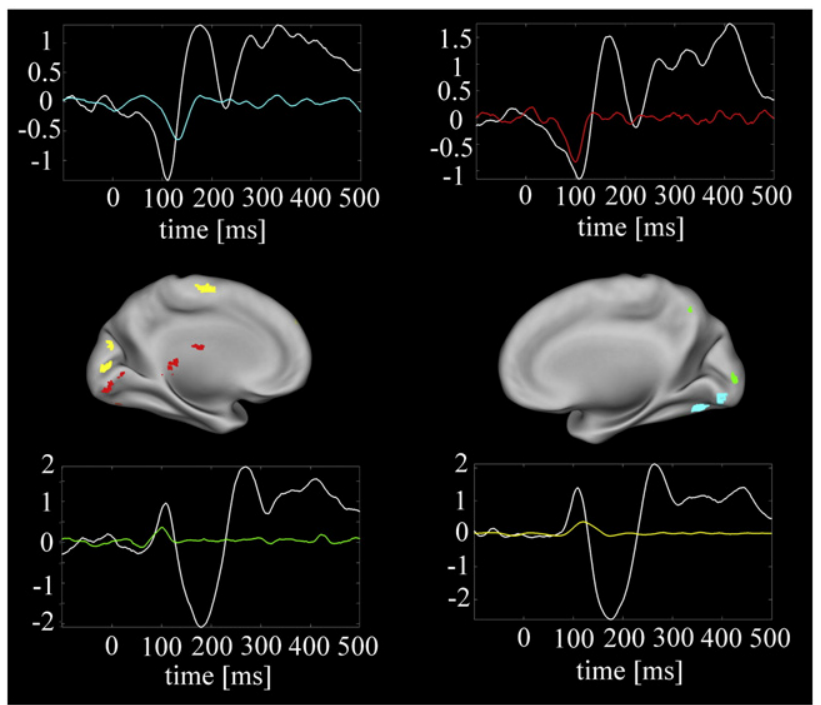

Fig. 3. Brain activations for the simultaneously recorded data, corresponding to the $\mathrm{C} 1$ ERP wave for all 4 different stimuli (extracted with the Oz electrode). The offset starts around $60 \mathrm{~ms}$, and the latency of the peak is around $100 \mathrm{~ms}$. The grand average ERPs (in white), and the ERP C1 ICs (color) of the particular stimuli are shown in the corners of the figure, such that their position describes the corresponding stimulus (i.e., the ERP corresponding to the upper left stimulus is presented in the upper-left corner of the figures, and the down-left, down-right and upper-right stimuli are presented in the counterclockwise order). In the middle of the figure, the functional activations are plotted on the medial view of the inflated brain. Left and right hemispheres are presented on the left- and right-hand side, respectively. Each functional activation is painted in the same color as the corresponding C1 ERP IC.

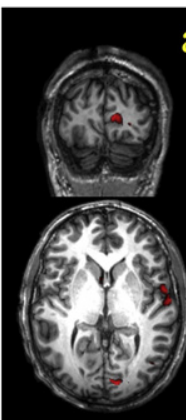

a

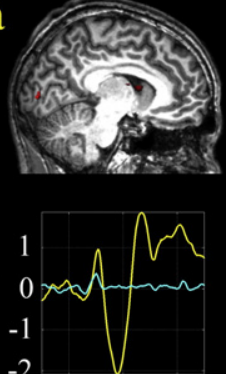

0200400
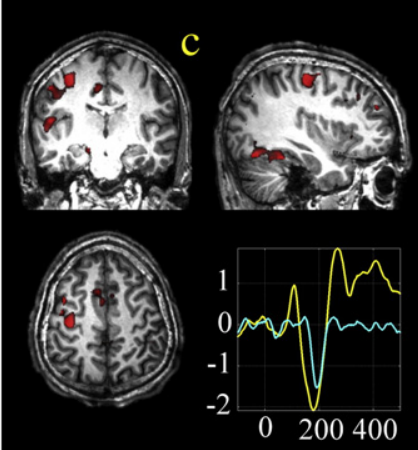

b
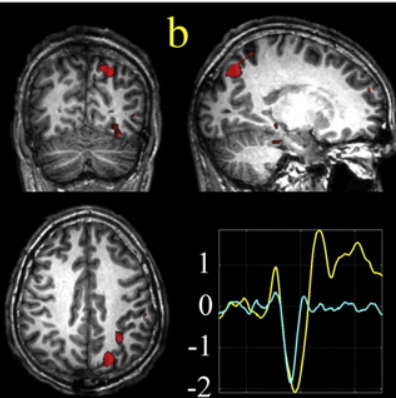

$0 \quad 200400$
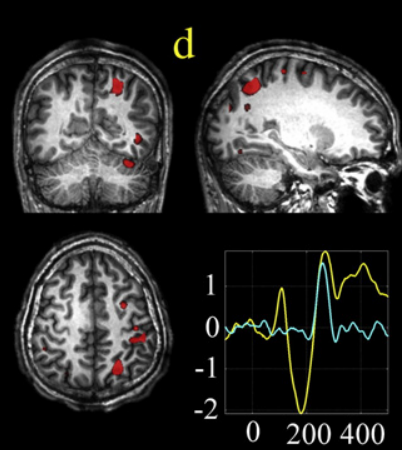

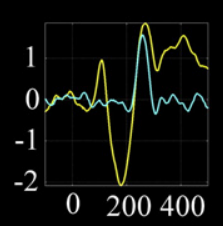

Fig. 4. The results of JointICA for the simultaneously recorded data from the Oz electrode for the down-left stimuli. Every extracted source contains an fMRI and an ERP part. Panel a shows the ERP wave containing the C1 peak. The corresponding fMRI activations are in the right upper lingual gyrus, around the calcarine sulcus - primary visual cortex (BA17, MNI [7 - 93 5]). In panel b, the early N1 component is present. The active fMRI areas are the right cuneus (BA7, MNI [18 - 72 46]) and right lingual gyrus (BA18, MNI [28, - $75-9]$ ). The late N1-related component (panel c), shows fMRI activations in the somatosensory cortex (BA3, 1 and 2) and primary motor cortex (BA4). The component in panel d shows P2 ERP activation, and the fMRI shows activity in the right cuneus (BA7, MNI [25 - 63 53]). shown in Fig. 5 are in strong agreement with the source localization that were obtained by Di Russo et al. (2003) using dipole-modeling.

That the outside data yield more activation than the inside ERP data is further evident from the fact that the results were obtained with only 18 datasets, or 8 less than in the analysis with data measured simultaneously. To further substantiate this, we repeated the jICA analysis with inside EEG data with only 18 datasets (the subset of subjects which has both inside and outside recordings, as mentioned before). The results of this analysis did not yield the expected P1-related fMRI activity (Fig. 7). This was contrary to the nonsimultaneous analysis in which the expected fMRI activation for the P1 wave was fully captured in the P1 IC (Fig. 5), despite that it included only 18 participants. This difference is probably due to residual scanner-related artifacts in the EEG data measured inside the scanner. Firstly, the quality difference of the ERPs recorded inside and outside the scanner room is obvious from Fig. 1 (especially from the prestimulus baseline variations), and from the SNR values provided in the Results section. Secondly, our previous work has shown that the quality of ERPs from EEG data recorded inside the scanner is strongly dependent on the procedures and parameter settings used for removing the artifacts and that the optimal settings vary from dataset to dataset (Assecondi et al., 2010; Vanderperren et al., 2010). Of course, given the differences in position, (neuro)physiology and measuring conditions between inside and outside EEG recordings, it cannot be excluded that some of the differences in the jICA result are also real differences that cannot be resolved with improved analysis and signal cleaning techniques.

Taking this precaution into account, we believe that the results reported here constitute a good argument for acquiring EEG and fMRI data in separate sessions, as it may yield better results with fewer participants. However, under certain circumstances separate recordings are not recommended. E.g., there are many cognitive studies in which processes, such as habituation, learning and arousal state (Debener et al., 2005, 2006), play an important role, and thus simultaneous measurements have to be performed. Also in some applications in clinical studies, simultaneous recordings are required. In both cases, it is essential to carefully optimize the data quality (e.g., Vanderperren et al., 2010) prior to further analyses.

\section{JointICA experiment - investigating the influence of the ICA algorithm}

Even though we know now which data properties are important to retrieve interpretable ICA components, this does not yet explain why exactly the JointICA method is able to extract these specific multimodal components. If one would consider general ICA assumptions, obtaining these components does not seem logical for the following reasons. First of all, the fMRI spatial activation maps in the brain have no mathematical ground to be fully statistically independent from each other (although this hypothesis is hard to check). This is why algorithms based on e.g. the diagonalization of 4 th order cumulant matrices (like JADE), and therefore destruction of all the 4th order cross-cumulants between the obtained independent components, are not able to separate fMRI sources correctly. This behavior was shown previously (Calhoun et al., 2001; Correa et al., 2007; Esposito et al., 2002), and the same result is confirmed here in Table 1. Although JADE does connect some components in the reliable way, other components are either merged together, or the ERP IC peaks are connected with non-corresponding fMRI IC activations (according to the expected activations based on the literature). Second, there is no reason to assume that ERP temporal peak components and fMRI spatial independent maps are statistically dependent, and will therefore be extracted into single independent component. For the above mentioned reasons, JADE is not the good choice for the JointICA algorithm, and therefore Calhoun et al. (2006) used Infomax. 


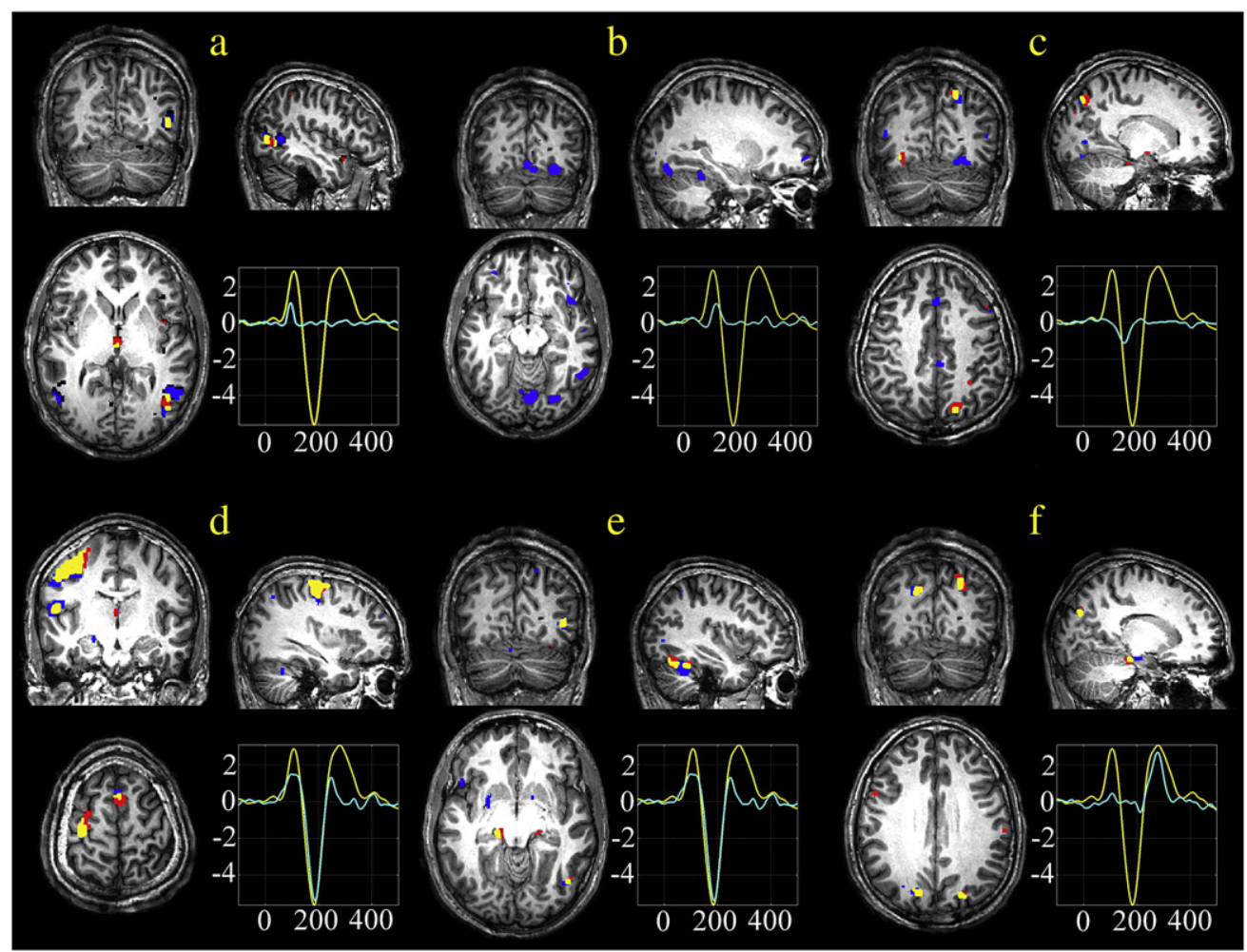

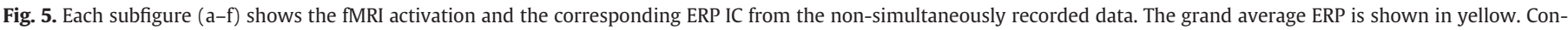

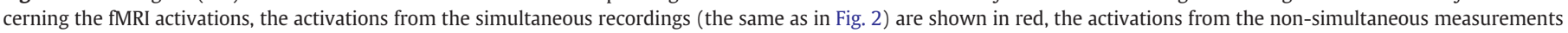

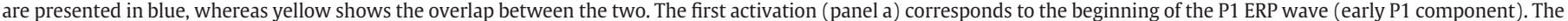

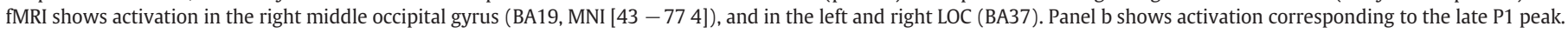

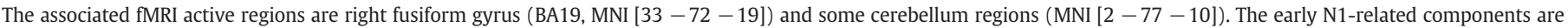

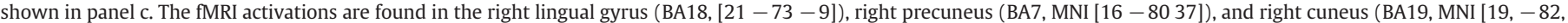

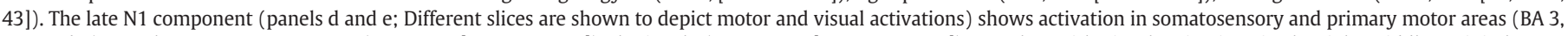

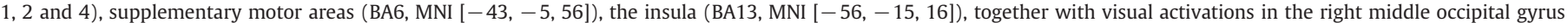
(BA19, MNI 43 - 77 4). The last, P2-related, component (panel f) shows activations in the left and right cuneus (BA19).

For the fMRI applications, the superior performance of Infomax, compared to other algorithms, has been shown by different groups (Calhoun et al., 2001, 2003; Daubechies et al., 2009; McKeown et al., 1998). Daubechies et al. (2009) showed on various simulations that the performance of Infomax is linked to its ability to effectively handle sparse components rather than independent components as such. FastICA algorithm has also been tested, and it showed more sensitivity to statistical independence of the data distributions with nonsparse data, but these distributions had no influence if the data used in the experiment were sparse. An explanation for this can be found in the fact that Infomax and FastICA are based on minimizing the nonlinear objective function, which was tailored to satisfy the expected distribution of the target components, not strictly implying statistical independence between components (Cardoso (1997) showed that it was possible to improve the Infomax estimates by tuning the nonlinearity to better match the underlying distributions). Specifically, since the data distribution of the target components was assumed to be super-Gaussian (sparse), a sigmoidal function is chosen for Infomax (McKeown et al., 1998), as implemented in the Fusion ICA Toolbox. For this reason, we expect Infomax to perform not only well on the fMRI but also on the joint data, assuming the sparsity of both modalities.

This means that, for Infomax to work, it is sufficient that brain areas corresponding to higher ERP peak amplitudes also need to show stronger hemodynamic responses and vice versa, and that the number of activated voxels with respect to the total number of analyzed voxels is small. If the amplitude modulation over subjects is linearly dependent between both modalities, due to Eq. (1) they will be captured in the same row of the $\mathbf{S}$ matrix, i.e., in the same independent component, even if the mutual independence between all the estimated components is not satisfied. As such, we can conclude that JADE is less effective in connecting fMRI activations with their corresponding ERP waveforms than FastICA or Infomax. For the analysis of the performance of the algorithms on the fMRI data, with a number of simulations where sparsity/independence was questioned, we refer the reader to Daubechies et al. (2009).

Concerning the FastICA algorithm, it pursues the same goal, using the concept of differential entropy (negentropy), again an information theoretic function, interpreted as a measure of non-Gaussianity of a distribution. FastICA practically estimates negentropy, based on maximum entropy principles (Hyvärinen, 1998). This results in the use of nonlinearities that are practically equivalent to those required by Infomax. Hence the decomposition by these algorithms is almost indistinguishable when 26 subjects are used (see the correlations in Table 1).

The difference between the two algorithms is the complementary way of adapting the unmixing matrices within each iteration. FastICA uses the non-adaptive rule, whereas Infomax uses the adaptive rule. The adaptation additionally requires the choice of a learning rate sequence, thereby affecting the convergence speed. However, the adaptation may account for some non-stationary effects in the observed data, and is more robust to the choice of the initial point (as confirmed by the ICASSO analysis in our work (Fig. 6)). For a more theoretical explanation about the algorithms and methods the reader is directed to (Infomax - Bell and Sejnowski, 1995; Fixed-Point approach Hyvärinen et al., 2001; Comon, 1994). 


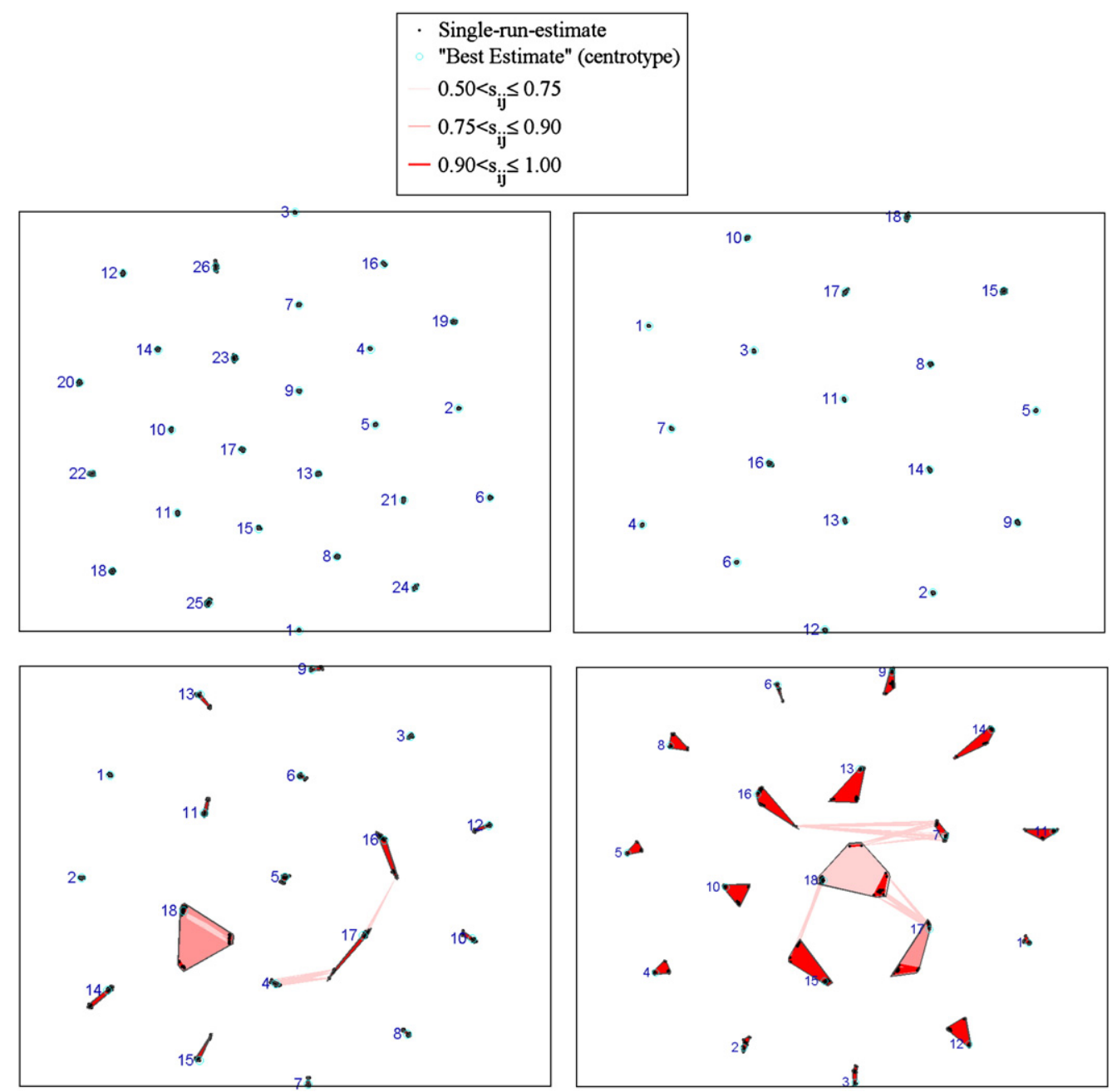

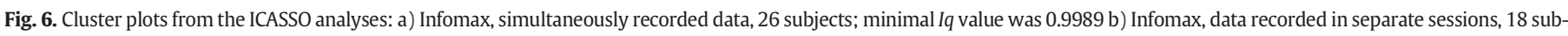

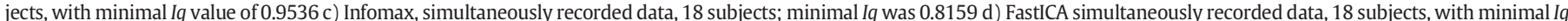

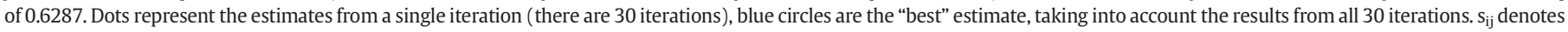
the correlation between the estimates. (For interpretation of the references to color in this figure legend, the reader is referred to the web version of this article.)

\section{JointICA experiment - central linking hypothesis and randomization}

To support our central-linking hypothesis that the strengths of activations in ERP and fMRI data are indeed highly correlated, we refer to work of Sadeh et al. (2010) for visual brain activation in humans. They examined the correlations between ERP and fMRI measures in face-selectivity. What they found is that not only face-selective ERP and fMRI responses were highly-correlated, but also that these correlations were specific for distinct ERP latencies and distinct brain regions. Following the same logic, we believe that these findings hold for all brain activations and that different ERP peak components are highly connected to different fMRI regions. This was also confirmed in a similar study with auditory stimulation by Mayhew et al. (2010) and in a visual study of Bagshaw and Warbrick (2007).

In the context of the central-linking hypothesis, we were comparing the similarity of the components obtained from integrated and individual ERP and fMRI ICA analyses. Based on this, and on the better results obtained with the non-simultaneously compared to the simultaneously acquired data (Figs. 5 and 7), we hypothesize that the JointICA decomposition is more strongly influenced by the ERP characteristics. It therefore represents an additional argument for optimizing the quality of the ERP data.

Nonetheless, the decomposition of the single modality ERP data was still significantly different from what was obtained in the joint decomposition. This is evident from the fact that some of the peaks obtained in distinct components in the separate analysis, are merged in the joint analysis, thanks to the fMRI maps. This indicates that, although the ERP data are more similarly decomposed, the fMRI modality also influences the decomposition significantly, which additionally confirms the central linking hypothesis.

As mentioned in the results, only $60 \%$ of the fMRI in the simultaneously processed data was explained through the P08 ERP components in Fig. 2, whereas additional 20\% is explained through a single fMRI independent component that shows activations in the primary visual areas, but has no ERP activation on the PO8 electrode. This fact is very interesting, since it implies that the so-called ERP-blind fMRI activations can be captured with the ICs having flat ERP part.

However, if the $\mathrm{Oz}$ electrode is used for decomposition, this same fMRI IC is connected to the C1 ERP IC. Therefore, the central linking theorem is additionally supported in the sense that a major part of the fMRI signal energy (more $80 \%$ in total) can indeed be explained with the ERP activity through the JointICA method. Moreover, it implies that the decomposition is more driven with the ERP modality, and explains the difference in the fMRI maps extracted jointly with the ERP data and in a separate analysis.

Another way to picture the central-linking hypothesis was the randomization analysis. It is shown that the ICs yield very low correlations with the randomized data (Table 3 ), significantly worse compared to the correlation coefficients obtained by comparison of Infomax to JADE (Table 1), or Infomax decomposition of solely the 


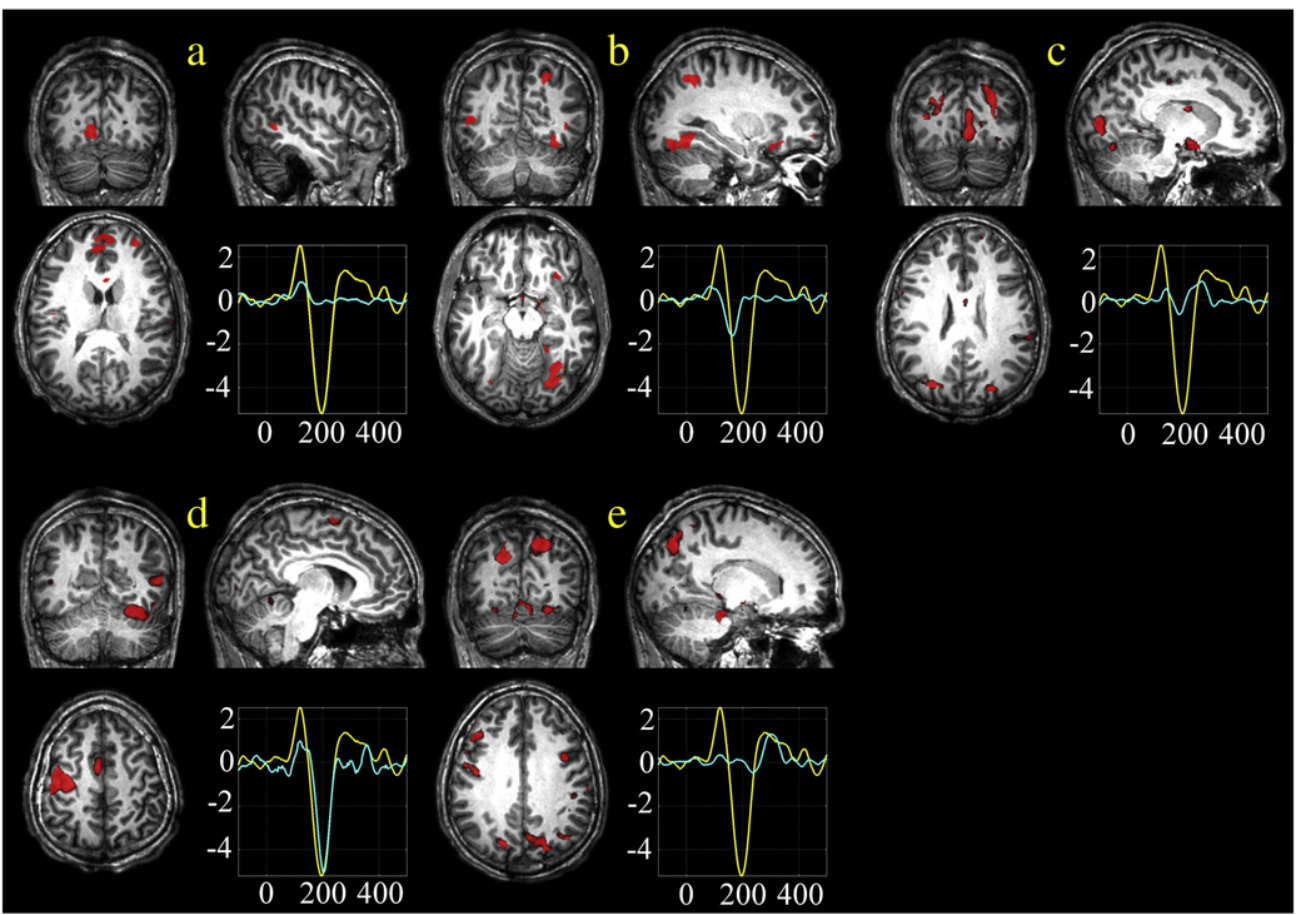

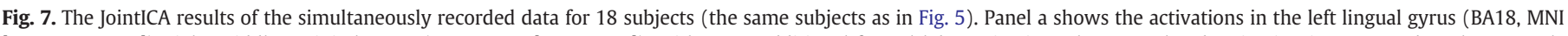

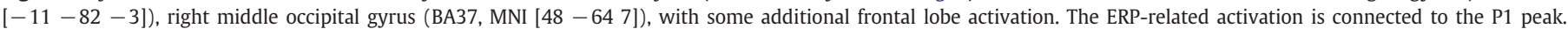

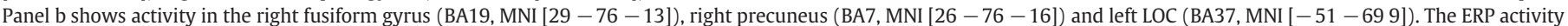

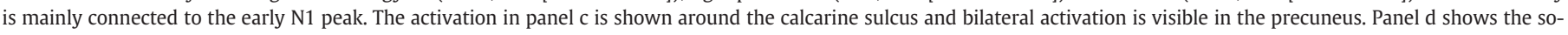

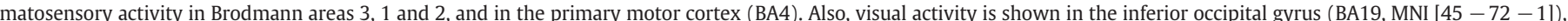

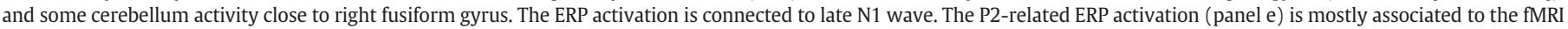
activation in the right and left precuneus (BA7).

fMRI data (Table 2). Additionally, when the data are randomized, the fMRI IC maps are connected to the wrong ERP independent components, or show no interpretable activation at all. This leads to the conclusion that the decomposition with randomized ERPs is worse than if no ERP information is used.

An additional indication that the ERP data are important for the algorithm, is given by the above-mentioned fact that different electrodes yield a different decomposition. This is shown in Figs. 2 and 4, visualizing the decompositions using electrodes $\mathrm{PO} 8$ and $\mathrm{Oz}$ respectively. However, these decompositions differ only in the early ERP components. When $\mathrm{Oz}$ is used, the fMRI IC shows activations around the calcarine sulcus (primary visual area), whereas if the PO8 electrode is used, the middle occipital gyrus (P1-related region) is activated. All the other activations (early N1, late N1 and P2) are similar between the two decompositions. The differences correspond well with the fact that the activations around the calcarine sulcus are connected with the C1 peak, which is visible on the midline electrodes (e.g., Oz), whereas the activations in the middle occipital gyrus give rise to the P1 peak that temporally overlaps with the $\mathrm{C} 1$, but is visible on the more lateral EEG electrodes (e.g., PO8). For more information about the expected occipital activations connected to the task used in this study, the reader is referred to Martinez et al. (2001) and Di Russo et al. (2002, 2003, 2005).

\section{JointICA experiment - robustness and consistency analysis}

Our results show that increasing the number of subjects has a clear impact on the quality of the decomposition. Table 1 shows that FastICA and Infomax extracted very similar independent

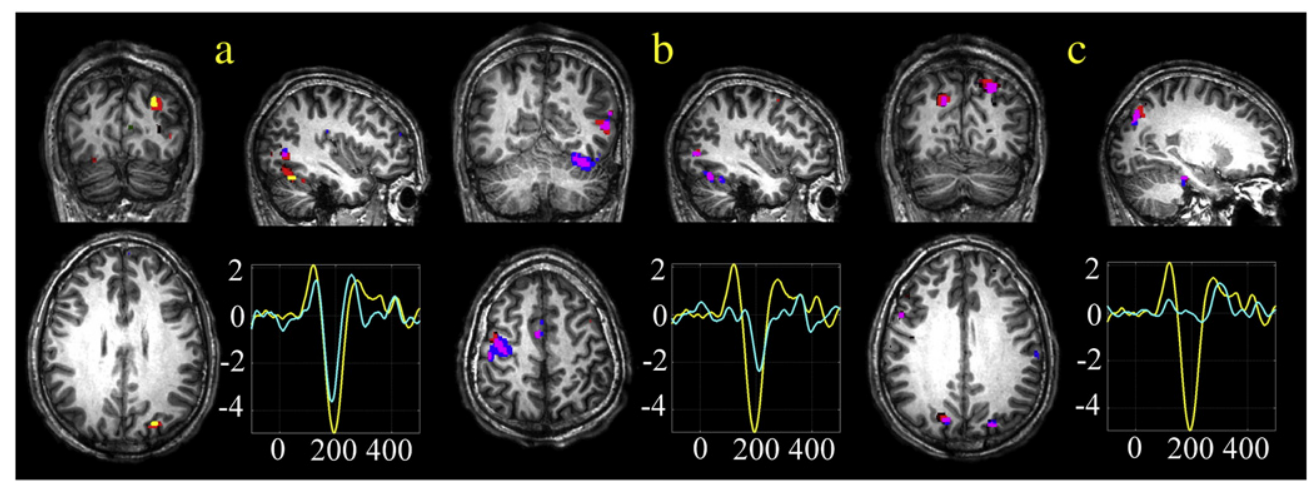

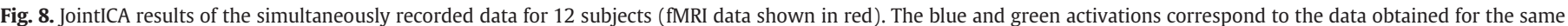

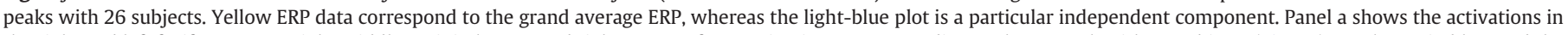

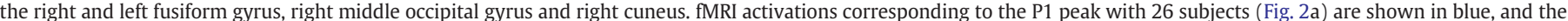

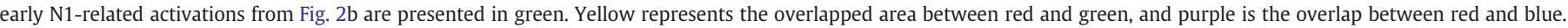

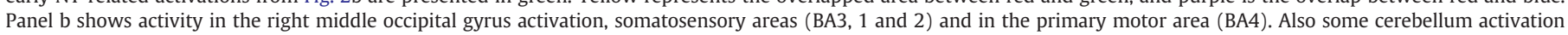
is visible in the vicinity of the fusiform gyrus. Panel c shows the fMRI IC activation in the left and right cuneus. 
components. Therefore, we conclude that, if the dataset is sufficiently large, both algorithms are going to show a consistent performance and yield similar results (as explained in one of the previous subsections). If the dataset is insufficient, the decomposition becomes unstable and Infomax performs better in the sense of consistency.

Fig. 7 (the decomposition of 18 subjects, simultaneously recorded data), teaches us that, apart for the P1-related independent component, other components are similar to when 26 subjects are used (Fig. 2). That implies (together with what we have seen by reducing the number of subjects from 26 to 18 ), that by taking less subjects into account, the decomposition is not going to change completely for all the components, but only for some (unstable) components, in this case P1. Other components are going to preserve their associated activations. This is because the information dimension is higher than the data dimension, and therefore it is no longer possible to separate the data into more independent components. This becomes even more obvious when the number of subjects drops to only 12 (Fig. 8).

As mentioned before, when JADE is used in JointICA, the ERP ICs and the fMRI IC maps are sometimes merged into one component, but sometimes also wrongly connected. The corresponding components in the Infomax decomposition with 12 subjects are only merged into one independent component. This is the consequence of a nonsufficient dataset. However, if more ERP ICs are merged into only one independent component, the corresponding fMRI ICs are also merged into the same component. Therefore, the decomposition can still be trusted.

Based on our research, we try to speculate on the test for the reliability of the decomposition. The decomposition can be considered reliable if it is found to be consistent (Iq above 0.9 ) systematically across consecutive numbers of subjects. This means that if the Iq value is high both in the experiment with $N$ subjects and in the experiment with $N+1$ or $N-1$ subjects, the decomposition is reliable. In that sense, reliable decompositions are shown in Figs. 2, 5 and 8, while the decomposition in Fig. 7 is not (see Table 5 for details). The consecutive number of subjects ensures the robustness of the decomposition. The consistency can be checked for both the Infomax and FastICA algorithms to ensure the sufficiency of the dataset.

However, as this claim is heuristically derived, it should only be taken as a hint for the researcher. Additional check, e.g. checking the existence of some physiologically expected components by an expert, should also be performed.

\section{Conclusion}

In this study, we investigated the performance of JointICA for EEG-fMRI data on a visual detection task. We showed and compared the performance when the EEG was recorded both simultaneously with fMRI data and in a separate session (outside the scanner room). The importance of the ICA algorithm, which is used for the decomposition, is also analyzed and discussed. Although it was previously suggested to use Infomax, it was better explained in the Discussion section of this manuscript why exactly this algorithm is the best choice. For this reason, its performance was also compared to FastICA and JADE. Additionally, we validated the central-linking hypothesis, and showed that the joint decomposition is mostly influenced by the ERP modality, and that therefore a good quality of the ERP data is highly desirable. It is also shown that the number of included subjects should be as high as possible, in order to obtain a complete decomposition. However, if the available number of subjects is not sufficient for the full decomposition, a meaningful decomposition can still be obtained, and we proposed a way to check if the obtained decomposition can be reliable. Although in this case some independent components are merged together, the corresponding ERP and fMRI activations still belong to the same independent component.

\section{Acknowledgments}

This research is supported by

- Research Council KUL: GOA MaNet, CoE EF/05/006 Optimization in Engineering (OPTEC), PFV/10/002 (OPTEC), IDO 05/010 EEG-fMRI, IDO 08/013 Autism;

- Flemish Government:

- FWO: PhD/postdoc grants, projects: FWO G.0302.07 (SVM), G.0341.07 (Data fusion), G.0427.10N (Integrated EEG-fMRI) research communities (ICCoS, ANMMM);

- TBM080658-MRI (EEG-fMRI);

$\circ$ IBBT

- Belgian Federal Science Policy Office: IUAP P6/04 (DYSCO, 'Dynamical systems, control and optimization', 2007-2011); ESA PRODEX No 90348 (sleep homeostasis).

JW is supported by long-term structural funding from the Flemish Government (METH/08/02).

MDV is supported by the Alexander Van Humbolt grant. The scientific responsibility is assumed by its authors.

\section{References}

Allen, P.J., Josephs, O., Turner, R., 2000. A method for removing imaging artifact from continuous EEG recorded during functional MRI. Neuroimage 12, 230-239.

Assecondi, S., Vanderperren, K., Novitskiy, N., Ramautar, J., Fias, W., Stiers, P., Sunaert S., Staelens, S., Van Huffel, S., Lemahieu, I., 2010. Effect of the static magnetic field of the MR-scanner on ERPs: evaluation of visual, cognitive and motor potentials. Clin. Neurophysiol. 121 (5), 672-685.

Bagshaw, A.P., Warbrick, T., 2007. Single trial variability of EEG and fMRI responses to visual stimuli. Neuroimage 38 (2), 280-292.

Bell, A.J., Sejnowski, T.J., 1995. An information-maximisation approach to blind separation and blind deconvolution. Neural Comput. 7 (6), 1129-1159.

Bénar, C.G., Grova, C., Kobayashi, E., Bagshaw, A.P., Aghakhani, Y., Dubeau, F., Gotman, J., 2006. EEG-fMRI of epileptic spikes: concordance with EEG source localization and intracranial EEG. Neuroimage 30 (4), 1161-1170.

Calhoun, V.D., Pearlson, G.D., 2004. Independent components analysis applied to FMRI data: a generative model for validating results. J. VLSI Sig. Proc. 37, 281-291.

Calhoun, V.D., Adali, T., Pearlson, G.D., Pekar, J.J., 2001. A method for making group inferences from functional MRI data using independent component analysis. Hum. Brain Mapp. 14, 140-151.

Calhoun, V.D., Adali, T., Hansen, L.K., Larsen, J., Pekar, J.J., 2003. ICA of functional fMRI data: an overview. Proc. Int. Workshop on ICA and BSS, pp. 281-288.

Calhoun, V.D., Adali, T., Pearlson, G.D., Kiehl, K.A., 2006. Neuronal chronometry of target detection: fusion of hemodynamic and event-related potential data. Neuroimage 30, 544-553.

Calhoun, V.D., Liu, J., Adali, T., 2009. A review of group ICA for fMRI data and ICA for joint inference of imaging, genetic, and ERP data. Neuroimage 45 (1 Suppl.) 163-172.

Calhoun, V.D., Wu, L., Kiehl, K.A., Eichele, T., Pearlson, G.D., 2010. Aberrant processing of deviant stimuli in schizophrenia revealed by fusion of fMRI and EEG data. Acta Neurpsychiatr. 22 (3), 127-138.

Cardoso, J.F., 1997. Infomax and maximum likelihood for source separation. IEEE Lett Signal Process. 4, 112-114.

Cardoso, J.F., Souloumiac, A., 1993. Blind beamforming for non-Gaussian signals. IEE Proc.-F 140 (3), 362-370.

Clark, V.P., Fan, S., Hillyard, S.A., 1995. Identification of early visually evoked potential generators by retinotopic and topographic analyses. Hum. Brain Mapp. 2 (170 18), 7.

Comon, P., 1994. Independent component analysis, a new concept? Signal Process. 36, 287-314.

Correa, N., Adali, T., Calhoun, V.D., 2007. Performance of blind source separation algorithms for fMRI using a group ICA method. Magn. Reson. Imaging 25, 684-694.

Correa, N.M., Li, Y.O., Adali, T., Calhoun, V.D., 2008. Canonical correlation analysis for feature-based fusion of biomedical imaging modalities and its application to detection of associative networks in schizophrenia. IEEE J. Sel. Topics Signal. 2 (6), 998-1007.

Correa, N., Li, Y.O., Adali, T., Calhoun, V.D., 2010. Canonical correlation analysis for data fusion and group inference. IEEE Signal Proc. Mag. 39, 39-50.

Czisch, M., Wetter, T.C., Kaufmann, C., Pollmacher, T., Holsboer, F., Auer, D.P., 2002. Altered processing of acoustic stimuli during sleep: reduced auditory activation and visual deactivation detected by a combined fMRI/EEG study. Neuroimage 16, 251-258.

Daubechies, I., Roussos, E., Takerkart, S., Benharrosh, S., Golden, C., D'Ardenne, K., Richter, W., Cohen, J.D., Haxby, J., 2009. Independent component analysis for brain fMRI does not select for independence. Proc. Natl. Acad. Sci. U. S. A. 106 (26), 10415-10422.

De Martino, F., de Borst, A.W., Valente, G., Goebel, R., Formisano, E., 2011. Predicting EEG single trial responses with simultaneous fMRI and relevance vector machine regression. Neuroimage 56 (2), 826-836. 
De Vos, M., Vergult, A., De Lathauwer, L., De Clercq, W., Van Huffel, S., Dupont, P., Palmini, A., Van Paesschen, W., 2007. Canonical decomposition of ictal scalp EEG reliably detects the seizure onset zone. Neuroimage 37 (3), 844-854.

De Vos, M., Ries, S., Vanderperren, K., Vanrumste, B., Alario, F.-X., Van Huffel, S., Burle, B., 2010. Removal of muscle artifacts from EEG recordings of spoken language production. Neuroinformatics 8 (2), 135-150.

Debener, S., De Vos, M., 2011. The benefits of simultaneous EEG-fMRI for EEG analysis. Clin. Neurophysiol. 122 (2), 267-277.

Debener, S., Ullsperger, M., Siegel, M., Fiehler, K., von Cramon, D.Y., Engel, A.K., 2005. Trial-by-trial coupling of concurrent electroencephalogram and functional magnetic resonance imaging identifies the dynamics of performance monitoring. J. Neurosci. 25 (50), 11730-11737.

Debener, S., Ullsperger, M., Siegel, M., Engel, A.K., 2006. Single-trial EEG-fMRI reveals the dynamics of cognitive function. Trends Cogn. Sci. 10 (12), 558-563.

Delorme, A., Makeig, S., 2004. EEGLAB: an open source toolbox for analysis of single-trial EEG dynamics including independent component analysis. J. Neurosci. Methods 134, 9-21.

Di Russo, F., Martinez, A., Sereno, M.I., Pitzalis, S., Hillyard, S.A., 2002. Cortical sources of the early components of the visual evoked potential. Hum. Brain Mapp. 15, 95-111.

Di Russo, F., Martinez, A., Hillyard, S.A., 2003. Source analysis of event-related cortica activity during visuo-spatial attention. Cereb. Cortex 13, 486-499.

Di Russo, F., Pitzalis, S., Spitoni, G., Aprile, T., Patria, F., Spinelli, D., Hillyard, S.A., 2005. Identification of the neural sources of the pattern-reversal VEP. Neuroimage 24, 874-886.

Doñamayor, N., Heilbronner, U., Münte, T.F., 2011. Coupling electrophysiological and hemodynamic responses to errors. Hum. Brain Mapp.. doi:10.1002/hbm.21305

Eichele, T., Calhoun, V.D., Moosmann, M., Specht, K., Jongsmae, M.L.A., Quian Quiroga R., Nordby, H., Hugdahl, K., 2008. Unmixing concurrent EEG-fMRI with parallel independent component analysis. Int. J. Psychophysiol. 67 (3), 222-234.

Esposito, F., Formisano, E., Seifritz, E., Goebel, R., Morrone, R., Tedeschi, G., Di Salle, F., 2002. Spatial independent component analysis of functional MRI time-series: to what extent do results depend on the algorithm used? Hum. Brain Mapp. 16, 146-157.

Franco, A.R., Ling, J., Caprihan, A., Calhoun, V.D., Jung, R.E., Heileman, G.L., Mayer, A.R., 2008. Multimodal and multi-tissue measures of connectivity revealed by joint independent component analysis. IEEE J. Sel. Topics Signal 2 (6), 986-997.

Goldman, R.I., Stern, J.M., Engel Jr., J., Cohen, M.S., 2000. Acquiring simultaneous EEG and functional MRI. J. Clin. Neurophysiol. 111, 1974-1980.

Goldman, R.I., Stern, J.M., Engel Jr., J., Cohen, M.S., 2002. Simultaneous EEG and FMRI of the alpha rhythm. Neuroreport 13, 2487-2492.

Himberg, J., Hyvärinen, A., 2003. Icasso: software for investigating the reliability of ICA estimates by clustering and visualization. IEEE Workshop on Neural Networks for Signal Processing, Touluse, France, pp. 259-268.

Himberg, J., Hyvärinen, A., Esposito, F., 2004. Validating the independent components of neuroimaging time-series via clustering and visualization. Neuroimage 22 (3), 1214-1222

Hyvärinen, A., 1998. New approximations of differential entropy for independent component analysis and projection pursuit. Adv. Neural Inf. Proc. Syst. 10, 273-279.

Hyvärinen, A., Karhunen, J., Oja, E., 2001. Independent Component Analysis. Wiley and Sons.

Krakow, K., Lemieux, L., Messina, D., Scott, C.A., Symms, M.R., Duncan, J.S., Fish, D.R. 2001. Spatio temporal imaging of focal interictal epileptiform activity using EEG triggered functional MRI. Epileptic Disord. 3 (2), 67-74.

Laufs, H., Kleinschmidt, A., Beyerle, A., Eger, E., Salek-Haddadi, A., Preibisch, C., Krakow, K., 2003. EEG-correlated fMRI of human alpha activity. Neuroimage 19, 1463-1476.

Lei, X., Qiu, C., Xu, P., Yao, D., 2010. A parallel framework for simultaneous EEG/fMRI analysis: methodology and simulation. Neuroimage 52 (3), 1123-1134.

Lei, X., Xu, P., Luo, C., Zhao, J., Zhou, D., Yao, D., 2011. fMRI functional networks for EEC source imaging. Hum. Brain Mapp. 32, 1141-1160.

Lemieux, L., Salek-Haddadi, A., Josephs, O., Allen, P., Toms, N., Scott, C., Krakow, K., Turner, R., Fish, D.R., 2001. Event-related fMRI with simultaneous and continuous EEG: description of the method and initial case report. Neuroimage 14 (3), 780-787.

Liebenthal, E., Ellingson, M.L., Spanaki, M.V., Prieto, T.E., Ropella, K.M., Binder, J.R., 2003. Simultaneous ERP and fMRI of the auditory cortex in a passive oddball paradigm. Neuroimage 19, 1395-1404

Liu, J., Calhoun, V., 2007. Parallel independent component analysis for multimodal analysis: application to fMRI and EEG data. Biomedical Imaging: From Nano to Macro, 4th IEEE International Symposium on, pp. 1028-1031.

Logothetis, N.K., Pauls, J., Augath, M., Trinath, T., Oeltermann, A., 2001. Neurophysiological investigation of the basis of the fMRI signal. Nature 412, 150-157.

Martinez, A., Di Russo, F., Anllo-Vento, L., Sereno, M.I., Buxton, B.B., Hillyard, S.A., 2001. Putting spatioal attention on the map: timing and localization of stimulus selection process in striate and extrastriate visual areas. Vision Res. 41, 1437-1457.

Martinez-Montes, E., Valdés-Sosa, P.A., Miwakeichi, F., Goldman, R.I., Cohen, M.S., 2004. Concurrent EEG/fMRI analysis by multiway partial least squares. Neuroimage 22 (3), 1023-1034
Mayhew, S.D., Dirckx, S.G., Niazy, R.K., Iannetti, G.D., Wise, R.G., 2010. EEG signatures of auditory activity correlate with simultaneously recorded fMRI responses in humans. Neuroimage 49 (1), 849-864.

McKeown, M.J., 2000. Detection of consistently task-related activations in fMRI data with hybrid independent component analysis. Neuroimage $11,24-35$

McKeown, M.J., Sejnowski, T.J., 1998. Independent component analysis of fMRI data: examining the assumptions. Hum. Brain Mapp. 6, 368-372.

McKeown, M.J., Makeig, A., Brown, G.G., Jung, T.P., Kindermann, S.S., Bell, A.J., Sejnowski, T.J., 1998. Analysis of fMRI data by blind separation into independent spatial components. Hum. Brain Mapp. 6, 160-188.

Moosmann, M., Ritter, P., Krastel, I., Brink, A., Thees, S., Blankenburg, F., Taskin, B., Obrig, H., Villringer, A., 2003. Correlates of alpha rhythm in functional magnetic resonance imaging and near infrared spectroscopy. Neuroimage 20, 145-158.

Moosmann, M., Eichele, T., Nordby, H., Hugdahl, K., Calhoun, V.D., 2008. Joint independent component analysis for simultaneous EEG-fMRI: principle and simulation. Int. J. Psychophysiol. 67 (3), 212-221.

Moosmann, M., Schonfelder, V.H., Specht, K., Scheeringa, R., Nordby, H., Hugdahl, K., 2009. Realignment parameter-informed artefact correction for simultaneous EEG-fMRI recordings. Neuroimage 45, 1144-1150.

Mulert, C., Lemieux, L., 2010. EEG-fMRI: Psychological Basis, Technique and Applications. Springer.

Mulert, C., Jäger, L., Schmitt, R., Bussfeld, P., Pogarell, O., Möller, H.J., Juckel, G., Hegerla, U., 2004. Integration of fMRI and simultaneous EEG: towards a comprehensive understanding of localization and time-course of brain activity in target detection. Neuroimage 22, 83-94.

Mulert, C., Seifert, C., Leicht, G., Kirsch, V., Ertl, M., Karch, S., Moosmann, M., Lutz, J., Moller, H.J., Hegerl, U., Pogarell, O., Jager, L., 2008. Single-trial coupling of EEG and fMRI reveals the involvement of early anterior cingulate cortex activation in effortful decision making. Neuroimage 42, 158-168.

Niazy, R.K., Beckmann, C.F., Iannetti, G.D., Brady, J.M., Smith, S.M., 2005. Removal of FMRI environment artifacts from EEG data using optimal basis sets. Neuroimage 28, 720-737.

Novitskiy, N., Ramautar, J.R., Vanderperren, K., De Vos, M., Mennes, M., Mijovic, B., Vanrumste, B., Stiers, P., Van den Bergh, B., Lagae, L., Sunaert, S., Van Huffel, S., Wagemans, J., 2011. The BOLD correlates of the visual P1 and N1 in single-trial analysis of simultaneous EEG-fMRI recordings during a spatial detection task. Neuroimage 54 (2), 824-835.

Porcaro, C., Ostwald, D., Bagshaw, A.P., 2010. Functional source separation improves the quality of single trial visual evoked potentials recorded during concurrent EEG-fMRI. Neuroimage 50 (1), 112-123.

Sadeh, B., Podlipsky, I, Zhdanov, A., Yovell, G., 2010. Event-related potential and functional MRI measures of face-selectivity are highly correlated: a simultaneous ERPfMRI investigation. Hum. Brain Mapp. 31 (10), 1490-1501.

Schabus, M., Dang-Vu, T.T., Albouy, G., Balteau, E., Boly, M., Carrier, J., Darsaud, A., Degueldre, C. Desseilles, M. Gais, S, Phillips, C. Rauchs, G, Schnakers, C, Sterpenich, V., Vandewalle, G., Luxen, A., Maquet, P., 2007. Hemodynamic cerebral correlates of sleep spindles during human non-rapid eye movement sleep. Proc. Natl. Acad. Sci. U. S. A. 104, 13164-13169.

Seeck, M., Lazeyras, F., Michel, C.M., Blanke, O., Gericke, C.A., Ives, J., Delavelle, J., Golay, X., Haenggeli, C.A., de Tribolet, N., Landis, T., 1998. Non-invasive epileptic focus localization using EEG-triggered functional MRI and electromagnetic tomography. Electroencephalogr. Clin. Neurophysiol. 106 (6), 508-512.

Ullsperger, M., Debener, S., 2010. Simultaneous EEG and fMRI, Recording, Analysis and Application. Oxford University Press, New York.

Vanderperren, K., De Vos, M., Ramautar, J.R., Novitskiy, N., Mennes, M., Assecondi, S. Vanrumste, B., Stiers, P., Van den Bergh, B.R.H., Wagemans, J., Lagae, L., Sunaert, S., Van Huffel, S., 2010. Removal of BCG artifacts from EEG recordings inside the MR scanner: a comparison of methodological and validation-related aspects. Neuroimage 50 (3), 920-934

Viola, F.C., Thorne, J., Edmonds, B., Schneider, T., Eichele, T., Debener, S., 2009. Semiautomatic identification of independent components representing EEG artifact. Clin. Neurophysiol. 120 (5), 868-877.

Wager, T.D., Nichols, T.E., 2003. Optimization of experimental design in fMRI: a general framework using a genetic algorithm. Neuroimage 18, 293-309.

Xu, L., Pearlson, G., Calhoun, V.D., 2009. Joint source based morphometry identifies linked gray and white matter group differences. Neuroimage 44 (3), 777-789.

Yang, L., Liu, Z., He, B., 2010. EEG-fMRI reciprocal functional neuroimaging. Clin. Neurophysiol. 121 (8), 1240-1250. 